\title{
Evolution of Periodic Orbits in the Sun-Saturn System
}

\author{
Niraj Pathak', R. K. Sharma' ${ }^{2}$ V. O. Thomas ${ }^{3}$ \\ ${ }^{1}$ Department of Mathematics, Dharmsinh Desai University, Nadiad, India \\ ${ }^{2}$ Department of Aerospace Engineering, Karunya University, Coimbatore, India \\ ${ }^{3}$ Department of Mathematics, Faculty of Science, The Maharaja Sayajirao University of Baroda, \\ Vadodara, India \\ Email: niraj23481@yahoo.com,ramkrishansharma@gmail.com,votmsu@gmail.com
}

Received 12 January 2016; accepted 12 June 2016; published 15 June 2016

Copyright (C) 2016 by authors and Scientific Research Publishing Inc.

This work is licensed under the Creative Commons Attribution International License (CC BY). http://creativecommons.org/licenses/by/4.0/

(c) (i) Open Access

\begin{abstract}
We analyze the periodic orbits, quasi periodic orbits and chaotic orbits in the photo gravitational Sun-Saturn system incorporating actual oblateness of Saturn in the planar circular restricted three body problem. In this paper, we study the effect of solar radiation pressure on the location of Sun centered and Saturn centered orbits, its diameter, semi major axis and eccentricity by taking different values of solar radiation pressure $q$ and different values of Jacobi constant " $C$ ", and by considering actual oblateness of Saturn using Poincare surface of section (PSS) method. It is observed that by the introduction of perturbing force due to solar radiation pressure admissible range of Jacobi constant $C$ decreases, it is also observed that as value of $C$ decreases the number of islands decreases and as a result the number of periodic and quasi periodic orbits decreases. Further, the periodic orbits around Saturn and Sun moves towards Sun by decreasing perturbation due to solar radiation pressure $q$ for a specific choice of Jacobi constant $C$. It is also observed that due to solar radiation pressure, semi major axis and eccentricity of Sun centered periodic orbit reduces, whereas, due to solar radiation pressure uniform change in semi major axis and eccentricity of Saturn centered periodic orbits is observed.
\end{abstract}

\section{Keywords}

Restricted Three Body Problem, Sun-Saturn System, Periodic Orbits, Quasi Periodic Orbits, Chaotic Orbits, Photo Gravitation, Oblateness, Poincare Surface Section

\section{Introduction}

Restricted three body problem (RTBP) describes the motion of an infinitesimal mass which moves under the 
gravitational effect of two finite masses called primaries. The primaries are supposed to move in circular orbits around their center of mass on account of their mutual attraction. Usually Sun and any one of its planets are taken as primaries. The secondary body is taken as the satellite of the primary planet or asteroid or comet or artificial satellite [1].

Lebedev experimentally demonstrated that the minute pressure exerted by light on bodies is inversely proportional to the square of the distance between the light source and the illuminated body. Since then many researchers have taken this force in to consideration, apart from other perturbing forces. [2] and [3] have shown that the role of the radiation force is rather complicated and its effect on the dynamics of the small body depends on its particular geometry, physical and physicochemical characteristics. Now the RTBP has widely ranging applications in the Solar system dynamics, the lunar theory, the motion of spacecrafts etc. "Theory of orbits" by [1] is one of the outstanding treatises on the RTBP. [4] have studied RTBP with all perturbation. He had considered the case where both primaries are source of radiation and both primaries and secondary bodies are oblate spheroids. He developed the equation of motion for two and three dimensional case with perturbation due to Coriolis and centrifugal forces. He identified location of equilibrium points, periodic orbits.

The study of periodic orbits plays an important role in the understanding of the general properties of different dynamical systems. A large number of periodic orbits were generated by [5] in the framework of RTBP using numerical techniques. [6] Surface of section (PSS) is widely used for analysing periodic, quasi periodic orbits and chaotic orbits. [7] have given a detailed analysis of periodic orbits using PSS. Chaotic behaviour of bodies can also be studied using PSS, Liapunov characteristic numbers, Fourier transform techniques and numerical irreversibility technique. The study of quasi-periodic orbits is important due to its application in space mission. One way of reducing fuel consumption is to place the spacecrafts on quasi-periodic orbits, thus maintaining a maximum separation. There have been numerous studies targeted at finding the quasi-periodic orbits around the libration points.

The set of stable periodic and quasi-periodic trajectories define regions of regular motion or stability "islands" that spread in a chaotic "sea" made of trajectories with high sensitivity with respect to the initial condition. As per Kolmogorov-Arnold-Moser (KAM) theory, the point represents a periodic orbit in the rotating frame, and the closed curves around the point correspond to the quasi-periodic orbits.

PSS gives a qualitative picture of stability regions in the planar problems. [8], employed multiple PSS method to find quasi-periodic orbits around the libration points $\mathrm{L}_{1}$ and $\mathrm{L}_{2}$ in the Sun-Earth system. [9] studied the location and stability of periodic and quasi-periodic orbits in the Earth-Moon system. [10] and [11] analysed the PSS for Earth-Moon system and Sun-Mars system. They have identified periodic, quasi-periodic solutions and chaotic regions. [12] and [13] also studied PSS for Saturn-Titan system for periodic orbits, quasi-periodic and chaotic regions.

In this paper we have studied PSS method for Sun-Saturn system for periodic and quasi-periodic orbits. This work is mainly concentrated on two major islands, one gives Sun centered orbit and other gives Saturn centered orbit. Since these two islands are major islands they are available in each PSS corresponding to different solar radiation pressure q with different Jacobi constant C. So, effect of perturbation on two different family of periodic orbit can be analysed by obtaining PSS. This paper is organised as follows. The basic equations of motion incorporating the perturbed force due to radiation and oblateness is given in Section 2. The PSS method is described in Section 3. Computational techniques are used to obtain PSS and periodic as well as quasi-periodic orbits in Section 4 and conclusions of the study are presented in Section 5.

\section{Equation of Motion}

Restricted three-body problem describes the motion of an infinitesimal mass which moves under the gravitational effect of two finite masses called primaries. The primaries are moving in circular orbits around their centre of mass on account of their mutual attraction and the infinitesimal mass not influencing the motion of the primaries.

We consider the case when bigger primary (Sun) is source of radiation and smaller primary (Saturn) is oblate spheroid. We consider that the equatorial plane of the Saturn is coincident with the plane of the motion and study only the planar case.

As the solar radiation pressure force $F_{p}$ changes with the distance by the same law as the gravitational attraction force $F_{g}$ and acts opposite to it, it is possible to consider that the result of the action of this force leads 
to reducing the effective mass of the sun [8].

Thus, the sun's resultant force acting on the particle is

$$
F=F_{g}-F_{p}=F_{g}\left(1-\frac{F_{p}}{F_{g}}\right)=q F_{g}
$$

where $q=1-\frac{F_{p}}{F_{g}}$ is the mass reduction factor constant for the given particle. If we follow the notation and terminology of [1] and [14]

$$
n^{2}=1+\frac{3}{2} A_{2}
$$

where

$$
A_{2}=\frac{A E^{2}-A P^{2}}{5 R^{2}}
$$

Here, AE and AP represent equatorial and polar radii of Saturn and R is the distance between Sun and Saturn.

Choose the unit of mass equal to the sum of the primary masses, the unit of length is equal to their separation and the unit of time is such that Gaussian constant of gravitation is unity. The usual dimensionless synodic coordinate system Oxy is used to express this motion. The origin of this system is positioned on the center of mass of the primaries while the bigger and smaller primaries always lie on the $O x$ axis at $P(-\mu, 0)$ and at $Q(1-\mu, 0)$, respectively.

Following [15] the equations of motion of the infinitesimal mass are

$$
\ddot{x}-2 n \dot{y}=\frac{\partial \Omega}{\partial x}, \ddot{y}+2 n \dot{x}=\frac{\partial \Omega}{\partial y} .
$$

where,

$$
\Omega=\frac{n^{2}}{2}\left[(1-\mu) r_{1}^{2}+\mu r_{2}^{2}\right]+\frac{q(1-\mu)}{r_{1}}+\frac{\mu}{r_{2}}+\frac{\mu A_{2}}{2 r_{2}^{3}}
$$

Here,

$$
r_{1}^{2}=(x-\mu)^{2}+y^{2}
$$

and,

$$
r_{2}^{2}=(x+1-\mu)^{2}+y^{2}
$$

By integrating, we get,

$$
\dot{x}^{2}+\dot{y}^{2}=2 \Omega-C
$$

where, $C$ is the Jacobi constant of integration.

$$
C=n^{2}\left((1-\mu) r_{1}^{2}+\mu r_{2}^{2}\right)+2 \frac{q(1-\mu)}{r_{1}}+2 \frac{\mu}{r_{2}}+\frac{\mu A_{2}}{r_{2}^{3}}-\dot{x}^{2}-\dot{y}^{2} .
$$

\section{Poincare Surface of Section}

The Poincare surface of section (PSS) method is used for determining the regular or chaotic nature of the trajectory. The numerical method of PSS is used to generate orbits and to study the location and stability of orbits in various systems.

In order to determine the orbital elements of the infinitesimal mass at any instant it is necessary to know its position and velocity, which correspond to a point in a four dimensional phase space. For the PSS method, the equations of motion are integrated in $(x, y)$ variables using a Runge-Kutta Gill fourth order variable or fixed step-size integrator. The initial conditions are selected along the $x$-axis. By defining a plane, say $y=0$, in the resulting three dimensional space the values of $x$ and $\dot{x}$ can be plotted every time the particle has $y=0$, 
whenever the trajectory intersects the plane in a particular direction, say $\dot{y}>0$. This section is obtained by fixing a plane in the phase space and plotting the points when the trajectory intersects this plane in a particular direction. We have constructed PSS on the $x, \dot{x}$ plane. The initial values were selected along the $O x$-axis by using intervals of length 0.001 and for few cases 0.0001 . The magnitude of the velocity vector is determined from its functional dependence on the Jacobi constant. The fine discretization of positions along the $x$-axis guarantees a wide coverage of the phase plane since each trajectory, regardless of the complexity of its motion, has a unique path through the phase plane. By giving different value of Jacobi constant we can plot the trajectories, and then we can do analysis of orbits. According to Kolmogorov-Arnold-Moser (KAM) theory, if there are smooth and well defined islands, then the trajectory is likely to be regular and the islands correspond to oscillation around a periodic orbit. As the curves shrink down to a point, the point represents a periodic orbit. Any fuzzy distribution of points in the surfaces of section implies that the trajectory is chaotic. Figure 1 shows PSS for Jacobi constant $C=3.018$ without perturbation due to solar radiation pressure and periodic orbit lying at the center of the island. This periodic orbit is Saturn centered periodic orbit.

\section{Computational Technique}

For Sun-Saturn system the mass of sun $m_{1}=1.9881 \times 10^{30} \mathrm{~kg}, m_{2}=568.36 \times 10^{24} \mathrm{~kg}$. Thus, $\mu=m_{2} /\left(m_{1}+m_{2}\right)=$ 0.0002857696 . Also equatorial radius of Saturn is $60268 \mathrm{~km}$, polar radius of Saturn is $54,364 \mathrm{~km}$. and distance between sun and Saturn is $1433000000 \mathrm{~km}$. So, according to the formula, $A_{2}=\frac{A E^{2}-A P^{2}}{5 R^{2}}$, oblateness coefficient $A_{2}=6.59158 \times 10^{-11}$. We have explored the Sun-centered and Saturn-centered orbits in the Sun-Saturn system and variations in them due to solar radiation pressure. For different solar radiation pressure we have different range of Jacobi constant $C$. For each value of $q$, we can find maximum value of $C$ using equation (6), such that $\dot{y}^{2}$ is positive. In other words, we can find admissible value of $C$ such that velocity of infinitesimal mass is real. $q=0.9$ gives maximum value of Jacobi constant $C$ as 2.807 such that infinitesimal mass having real velocity within region between two primaries, Sun and Saturn. In other words, for $q=0.9$, admissible range of $C$ lie in the interval $[1,2.807]$ for values of $x$ in the interval $[0,1]$. If $C=2.808$ then for same $q, \dot{y}^{2}$ becomes negative for $x$ in the range [0.9290, 0.9510]. This is excluded region for infinitesimal mass as velocity becomes complex. For $q=0.9$, we have analysed the PSS for $1 \leq C \leq 2.8$ and $\Delta C=0.1$. PSS for $C=1.0$ contains only 2 points as shown in Figure 2. PSS from $C=1.0$ to $C=1.7$ contains only fuzzy distribution of points without any indication of islands which gives only chaotic orbits. PSS for $C=1.7$ is shown in Figure 3. Chaotic orbits for $C=$ 1.7, $q=0.9$ and $x=0.8032$ and time $t=500$ and for $C=1.7, q=0.9$ and $x=0.1269$ and time $t=500$ are shown in Figure 4 and Figure 5 respectively.

First quasi periodic orbit obtained at $x=0.1168$ for $C=1.8$ when $q=0.9$. This orbit is located at the center of the island as shown in Figure 6. Which is first Sun centered quasi periodic orbit when $q=0.9$.

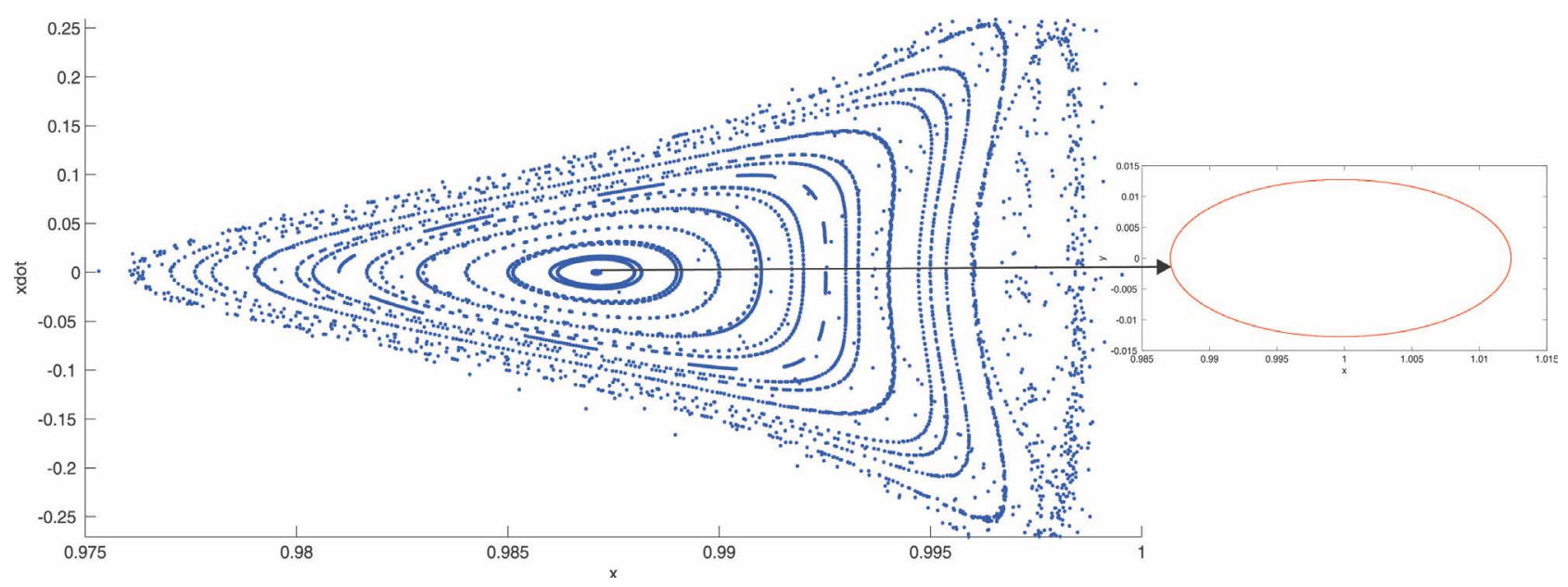

Figure 1. PSS for Jacobi constant $C=3.018, q=1$ and periodic orbit lying at the center of the island. 


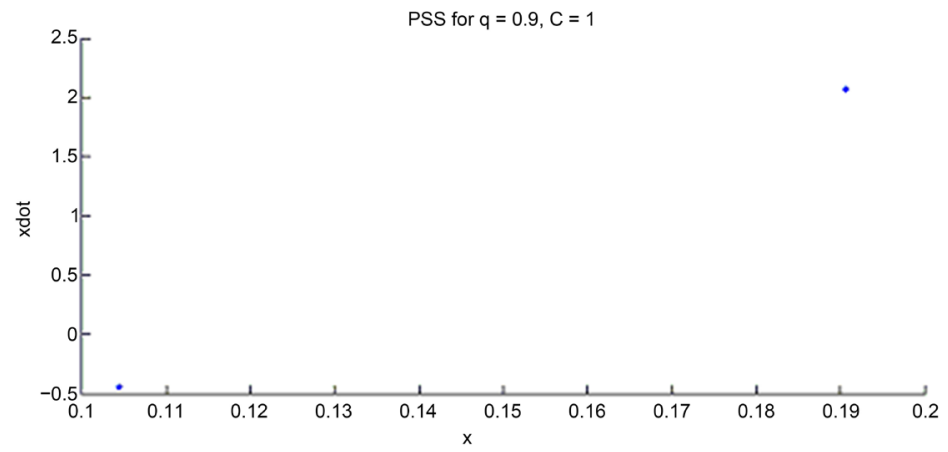

Figure 2. PSS for $C=1.0$ with $q=0.9$.

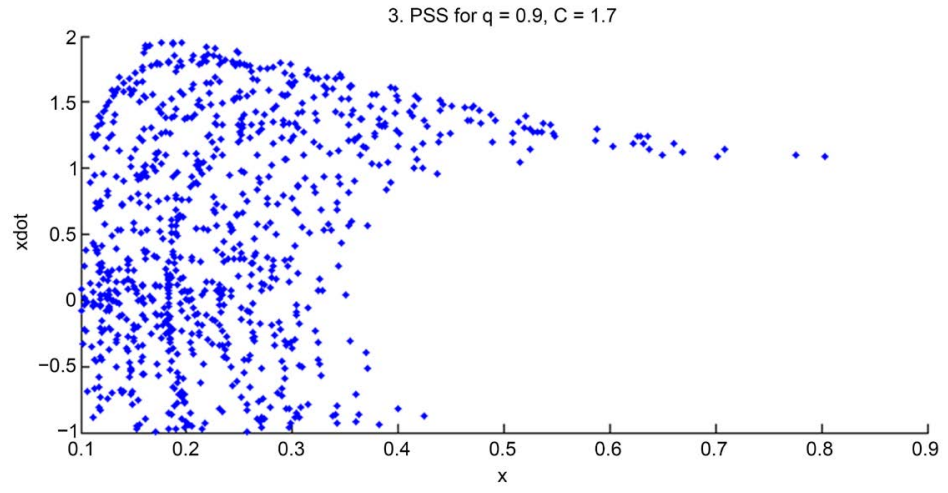

Figure 3. PSS for $C=1.7$ with $q=0.9$.

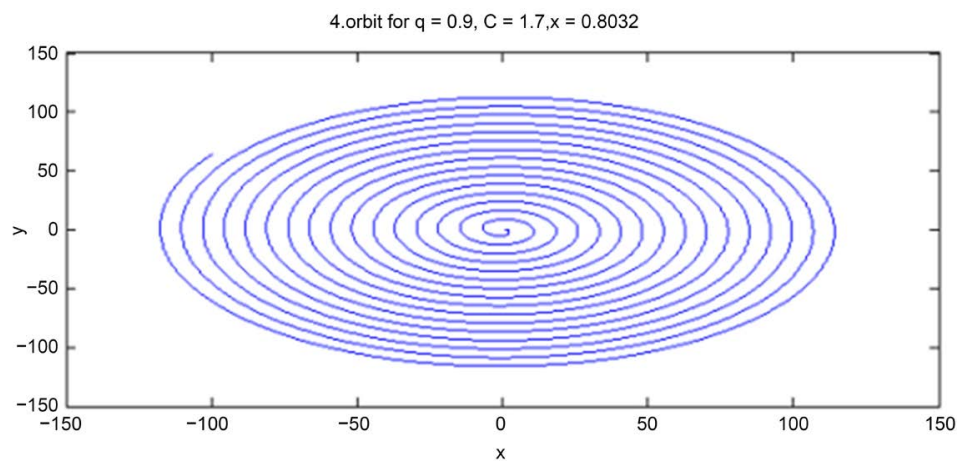

Figure 4. Orbit at $x=0.8032$ for $C=1.7, q=0.9$.

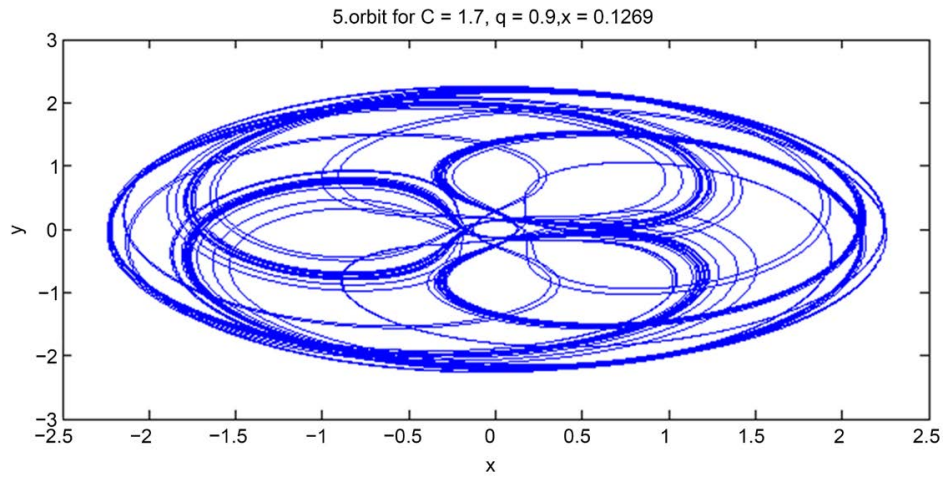

Figure 5. Orbit at $x=0.1269$ for $C=1.7, q=0.9$. 


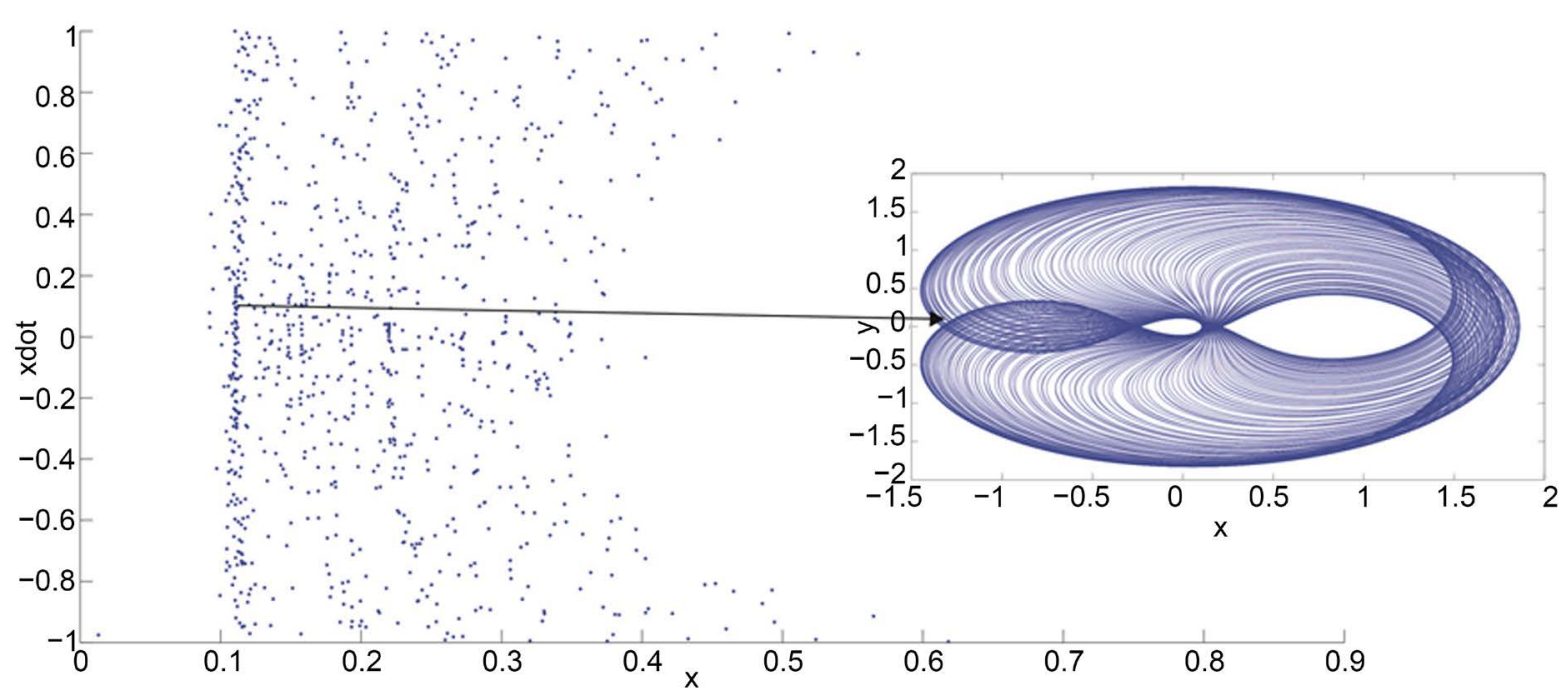

Figure 6. PSS for $C=1.8, q=0.9$ and orbit corresponding to point $x=0.1168, t=1000$.

By observing PSS for different values of Jacobi constants from $C=1.0$ to $C=2.8$ we conclude that as value of $C$ increases, the number of islands increases and as a result periodic orbits and quasi periodic orbits increase.

\subsection{Sun Centered Periodic Orbits}

Here, we mainly concentrate on one of the major island which gives sun centred orbit and analyze its nature for different Jacobi constant $C$.

Figure 7(a) and Figure 8(a) show PSS for $q=0.9$ with Jacobi constant $C=2.79,2.8$ respectively. Each PSS is formed between two primaries Sun and Saturn located at $x=0$ and $x=1$ respectively. Figure 7(b) and Figure 8(b) show enlarged view of the island on which study is focused. Figure 7(c) and Figure 8(c) show prograde Sun centered periodic orbit corresponding to the center of the island.

Figure 9 depicts variation in location of Sun centered periodic orbit with $C$ for $q=0.9$. It can be observed that as $C$ increases location of periodic orbit moves towards 1 . From analysis of one of the major island and Sun centered periodic orbit corresponding to center of the island when $q=0.9$, it is concluded that as $C$ increases location of periodic orbit moves towards Saturn.

Semi-major axis " $a$ " and eccentricity " $e$ " of the Sun centered orbit are obtained by Murraay and Dermott (1999) as

$$
\begin{gathered}
a=\left[\frac{2}{r_{1}}-\frac{V^{2}}{1-\mu}\right]^{-1} \\
e=\sqrt{1-\frac{h^{2}}{a(1-\mu)}} \\
V^{2}=\left(\dot{x}^{2}+(\dot{y}+x+\mu)^{2}\right) \\
h=\left(x+\mu_{2}\right)(y+\dot{x}+\mu) \\
r_{1}^{2}=(x+\mu)^{2}+y^{2}
\end{gathered}
$$

For Sun centered periodic orbits, Figure 10 and Figure 11 show that as Jacobi constant $C$ increases semi major axis increases and eccentricity decreases.

By reducing perturbation due to solar radiation pressure $(q=0.9845)$ using same procedure admissible range of Jacobi constant $C$ is $[1,2.985]$. $C=2.986$ gives negative $\dot{y}^{2}$ within region $x=0.948$ to $x=0.960$ which is excluded region for infinitesimal mass as velocity becomes complex. It is observed that excluded region is shifted towards Saturn. 


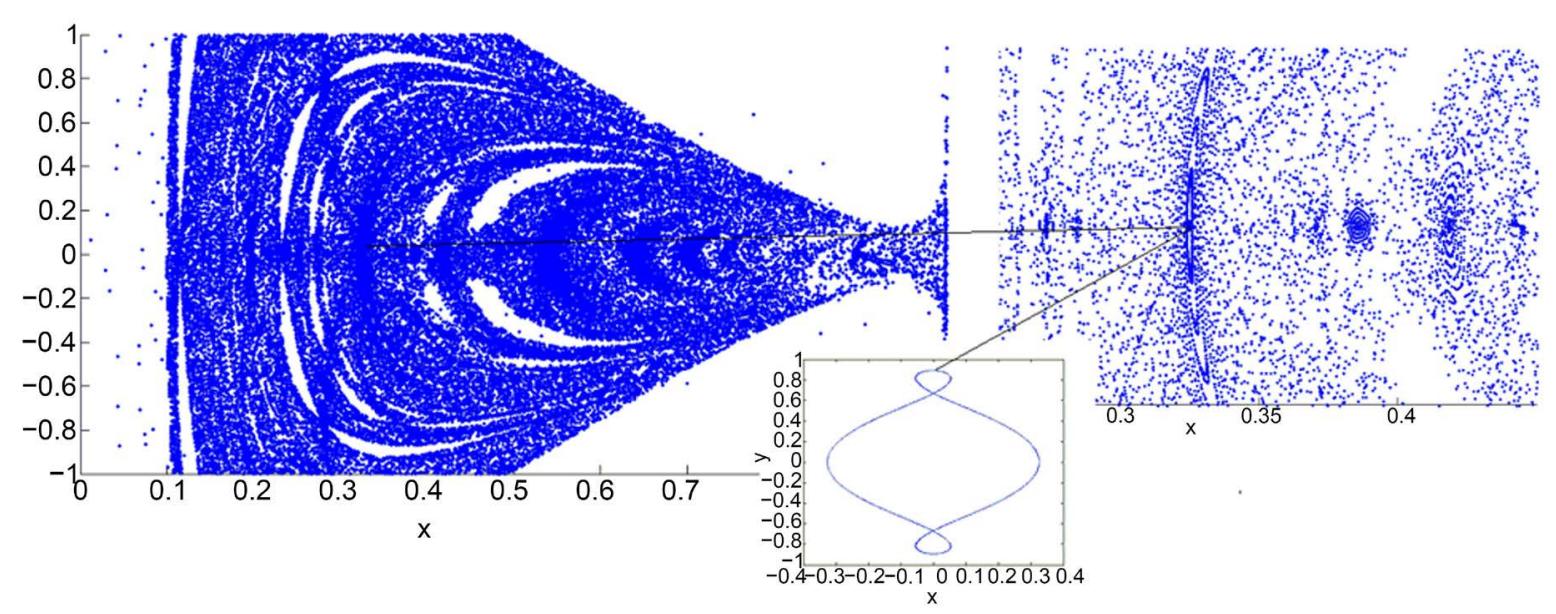

Figure 7. (a) PSS for $C=2.79$ with $q=0.9$, (b) island near $x=0.32$ and (c) center of the island showing Sun centered periodic orbit at $x=0.3249, t=1000$.

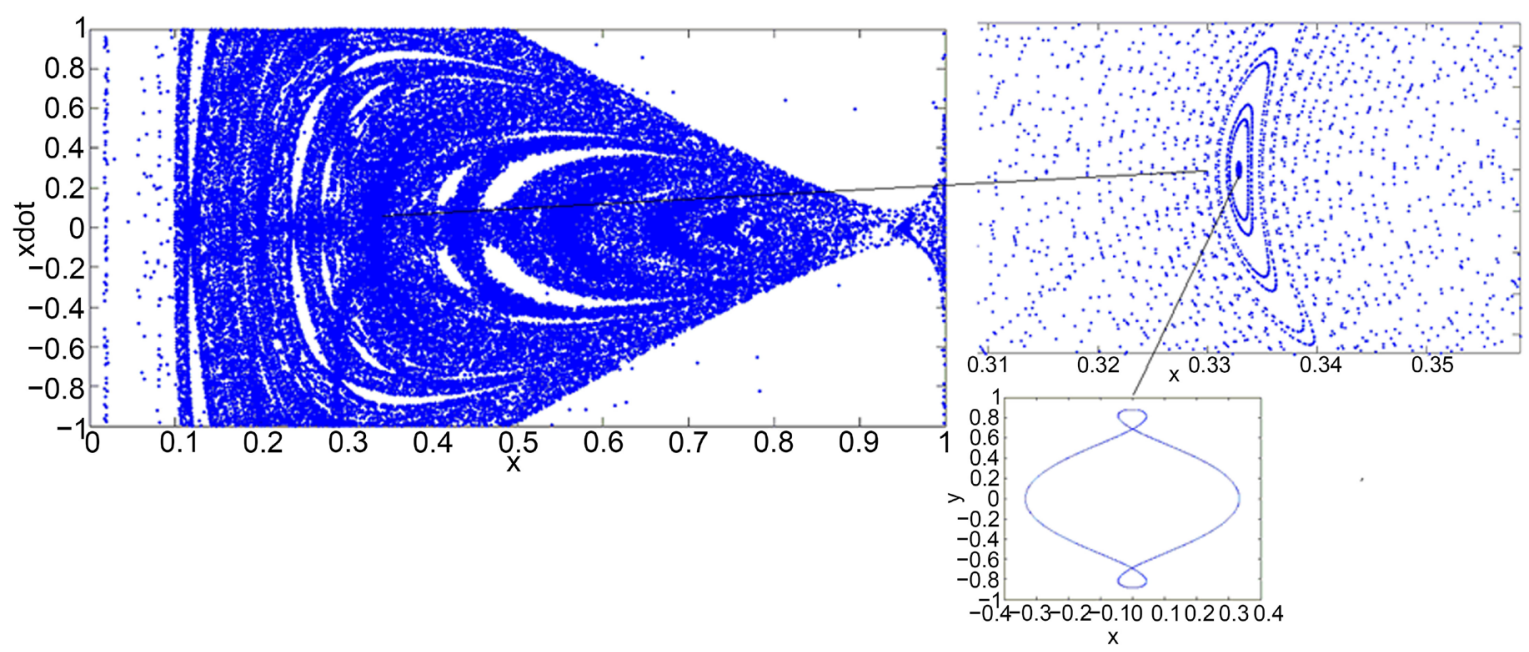

Figure 8. (a) PSS for $C=2.8$ with $q=0.9$; (b) island near $x=0.33$ and (c) center of the island showing Sun centered periodic orbit for $x=0.33286, t=1000$.

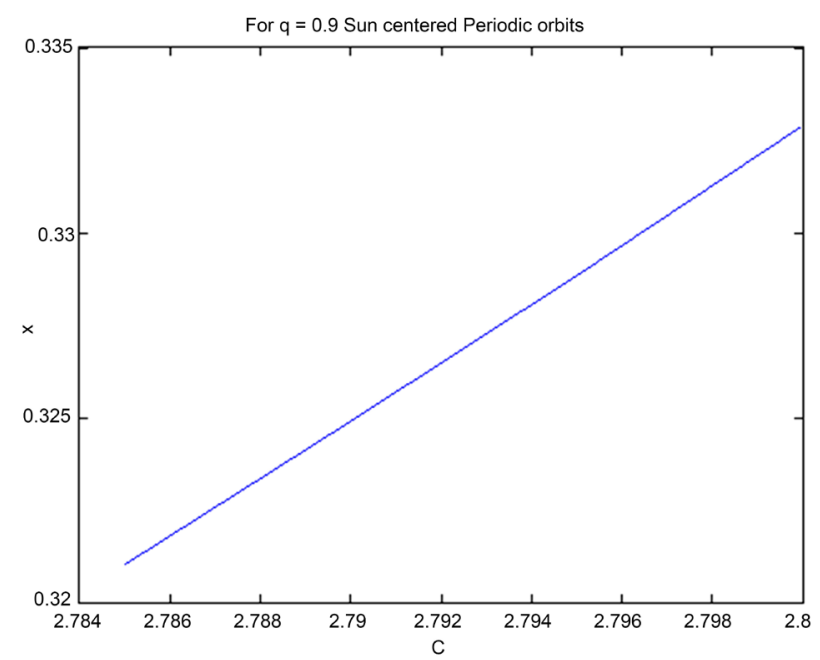

Figure 9. Position of periodic orbit vs Jacobi constant for $q=0.9$. 


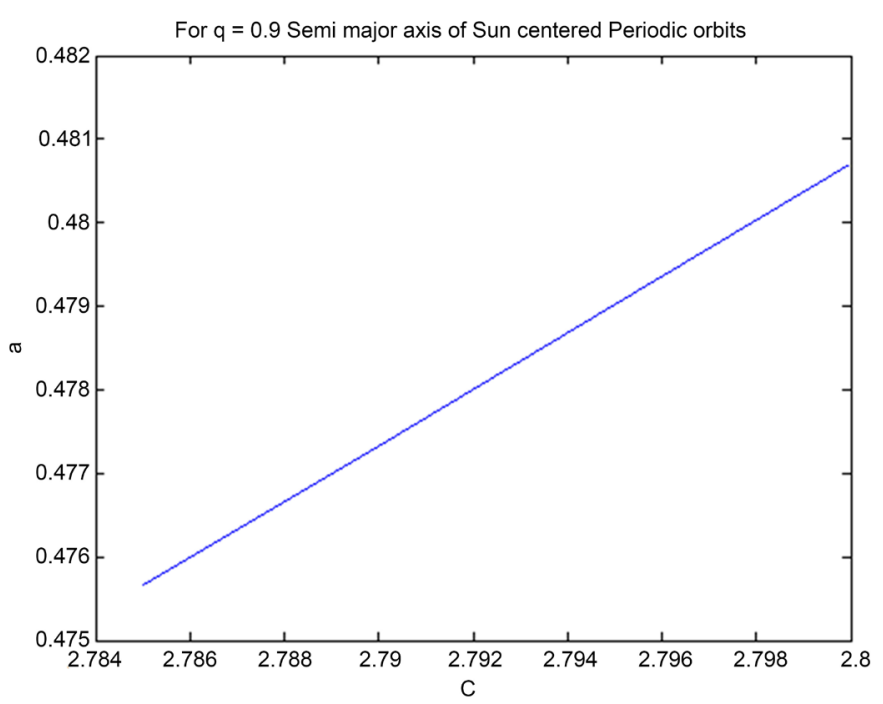

Figure 10. Variation in semi major axis for $q=0.9$.

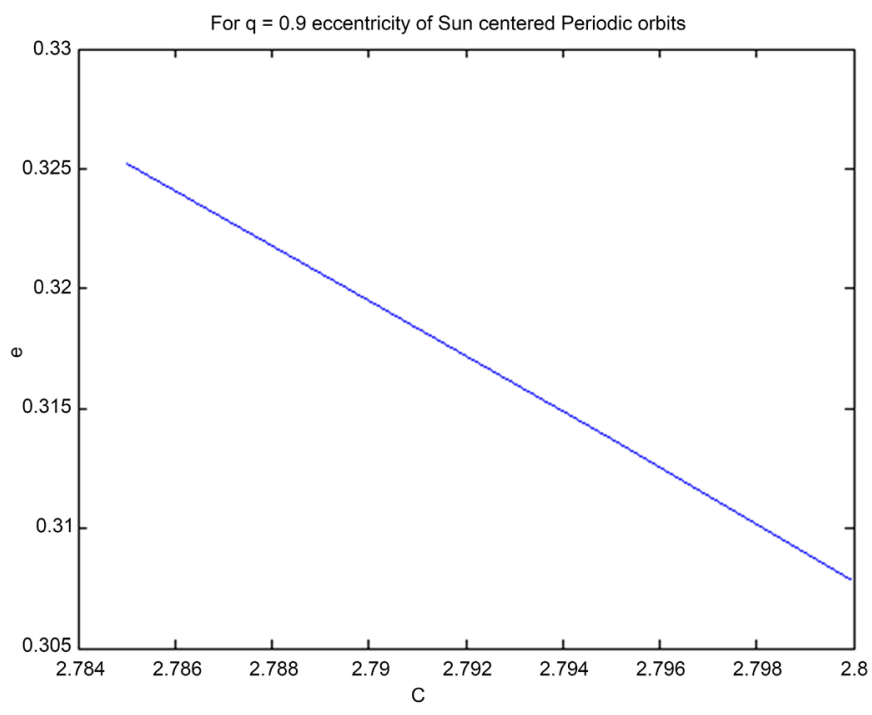

Figure 11. Variation in eccentricity for $q=0.9$.

Figure 12(a) and Figure 13(a) shows PSS for $\mathrm{q}=0.9845$ with Jacobi constant $C=2.985$ and 2.975 respectively. When we neglect the perturbation due to solar radiation pressure (i.e. $q=1)$ using same procedure admissible range of Jacobi constant $C$ is $[1,3.018]$. $C=3.019$ gives negative $\dot{y}^{2}$ within region $x=0.946$ to $x=0.964$ which is excluded region for infinitesimal mass as velocity becomes complex. Here it is observed that excluded region becomes larger. With reduced perturbation, analysis of same island and periodic orbit corresponding to its center gives conclusion that increment in value of Jacobi constant is responsible for shifting of location of periodic orbits towards Saturn which is shown in Figure 16.

For Sun centered periodic orbits, Figure 17 and Figure 18 shows that as Jacobi constant $C$ increases semi major axis orbit increases and eccentricity decreases. By neglecting the solar radiation pressure $(q=1)$ the maximum value of Jacobi constant increases up to 3.018. But for analyzing the effect of solar radiation pressure on Sun centered periodic we consider the same Jacobi constants as above.

For $q=1$, the shifting of periodic orbits towards Saturn by increasing value of Jacobi constant $C$ is shown in Figure 16. Figure 12(c) and Figure 14(c) show Sun centered periodic orbit for $C=2.985$ with $q=0.9845$ and $q=$ 1 respectively. Figure 14(c) and Figure 15(c) show Sun centered periodic orbit for $C=2.985$ and 2.975 and 2.97 with $q=1$. Effect of solar radiation pressure on Sun centered periodic orbit obtained for a given $C$. It is 


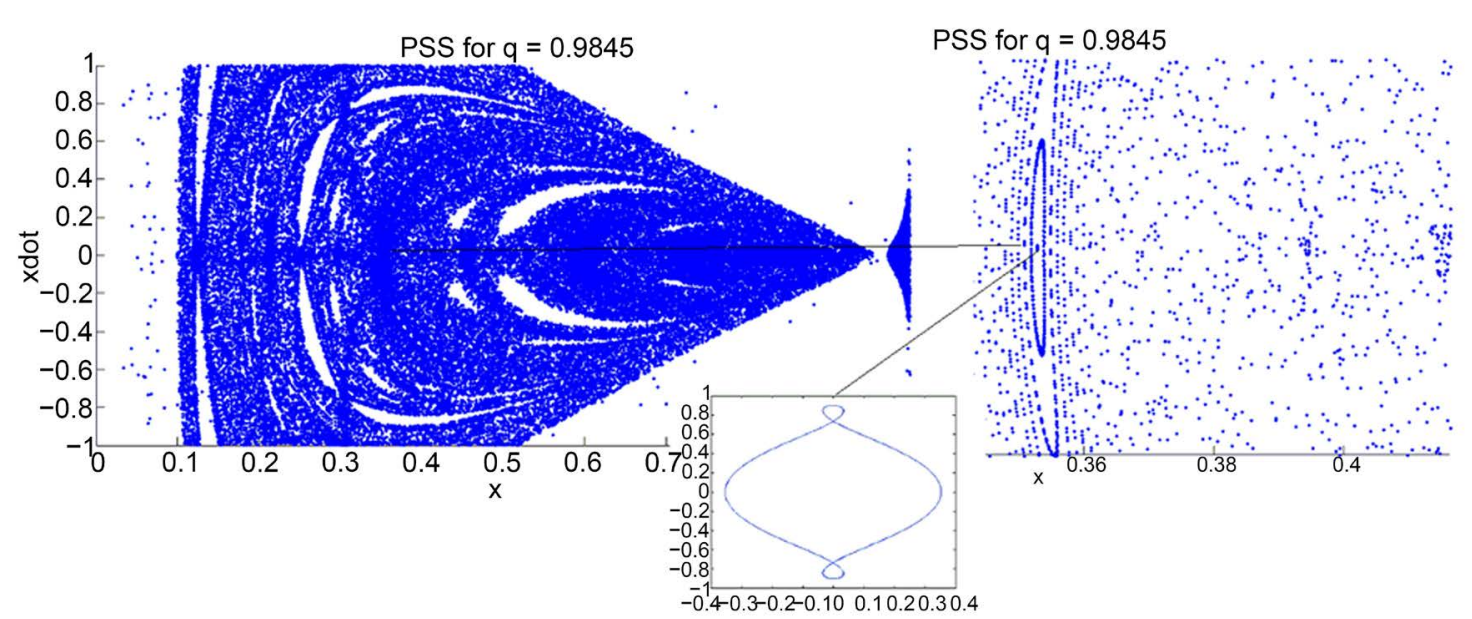

Figure 12. (a) PSS for $C=2.985$ with $q=0.9845$; (b) island near $x=0.355$ and (c) center of the island showing Sun centered periodic orbit for $x=0.352983, t=1000$.

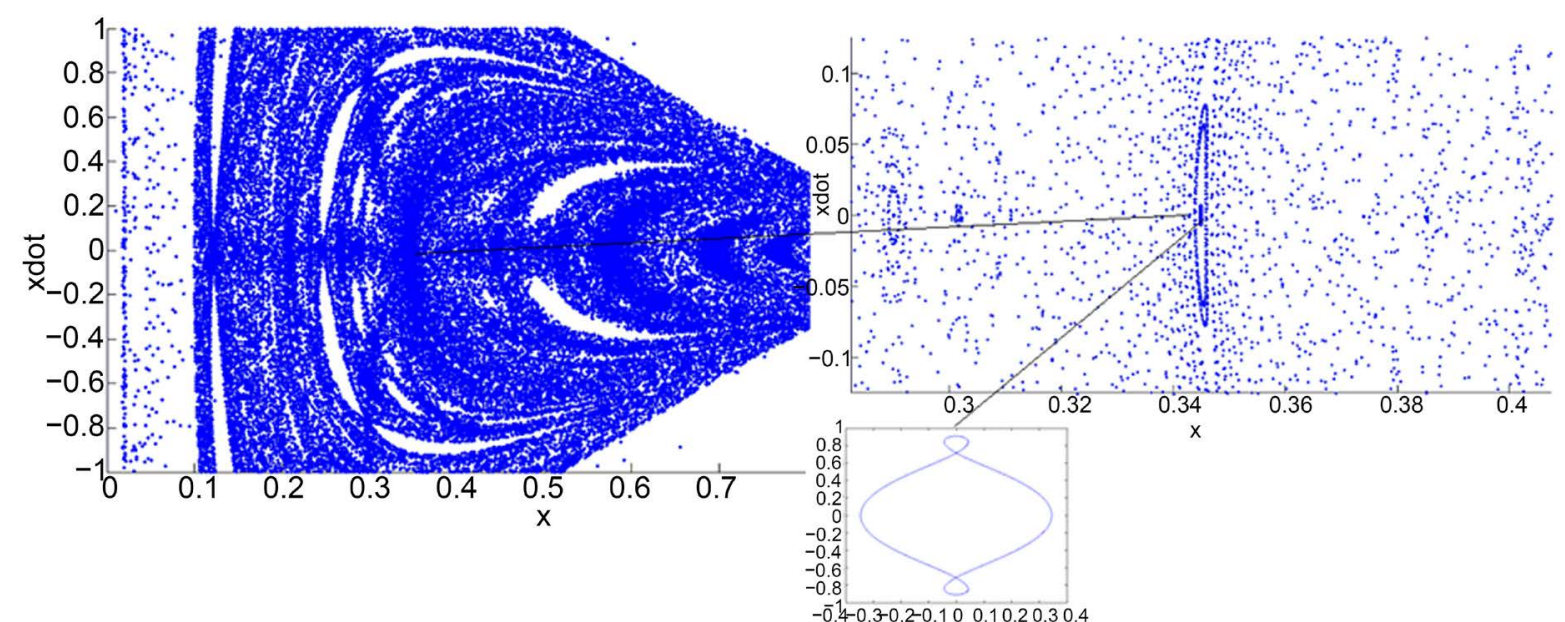

Figure 13. (a) PSS for $C=2.975$ with $q=0.9845$; (b) island near $x=0.34$ and (c) center of the island showing Sun centered periodic orbit for $x=0.3449, t=1000$.

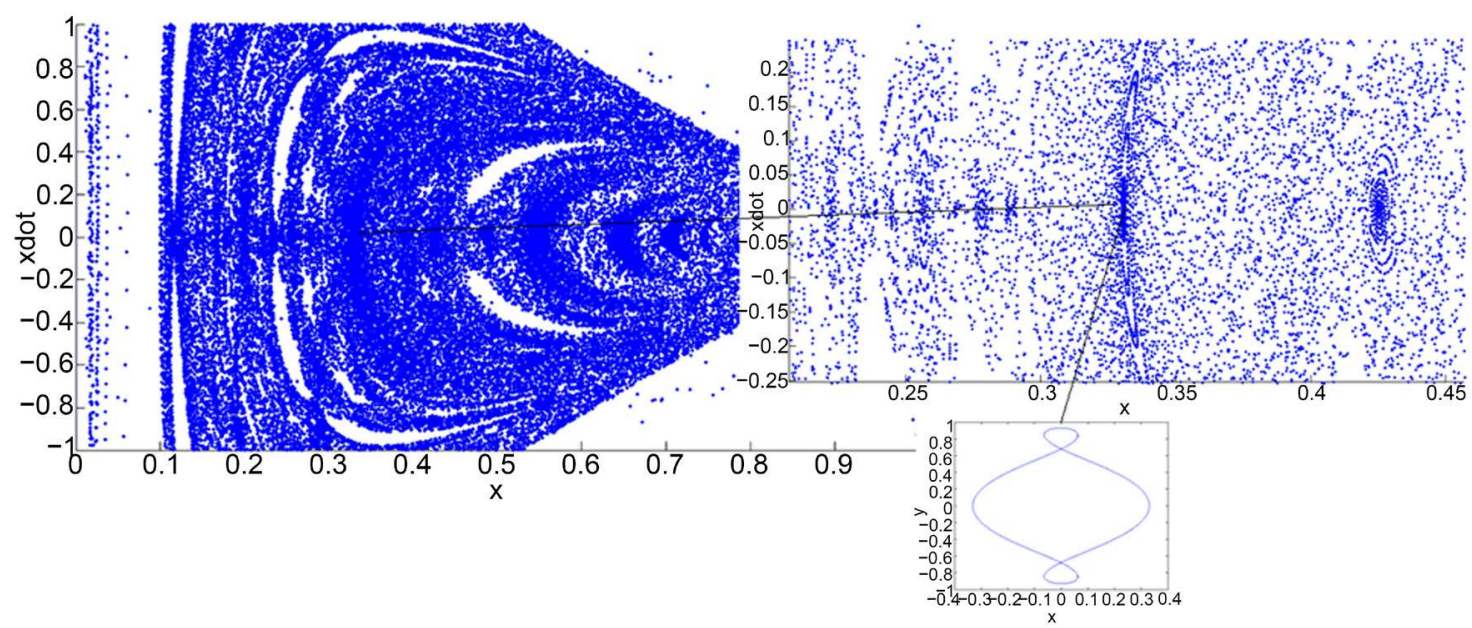

Figure 14. (a) PSS for $C=2.985$ with $q=1$; (b) island near $x=0.33$ and (c) center of the island showing Sun centered periodic orbit for $x=0.3306, t=1000$. 


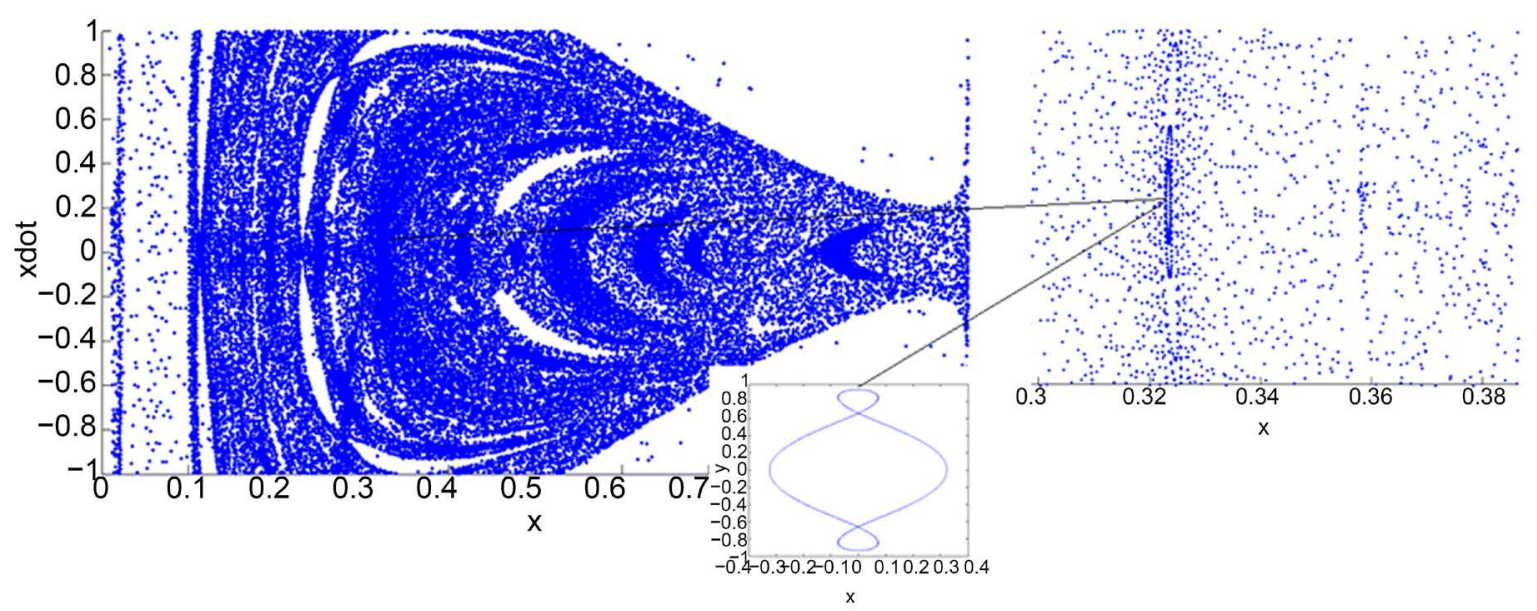

Figure 15. (a) PSS for $C=2.975$ with $q=1$; (b) island near $x=0.3235$ and (c) center of the island showing Sun centered periodic orbit for $x=0.32335, t=1000$.

concluded that solar radiation pressure is responsible for shifting location of periodic orbits towards Saturn which is shown in Figure 16.

It is observed from Figure 17 that as Jacobi constant $C$ increases semi major axis increases. It is clearly observed that as perturbation due to solar radiation pressure decreases semi major axis of periodic orbits for same Jacobi constant $C$ increases.

Figure 18 shows that as Jacobi constant $C$ increases, eccentricity decreases. It is concluded that solar radiation pressure is responsible for reducing eccentricity of Sun centered periodic orbit for same $C$.

\subsection{Saturn Centered Periodic Orbits}

By considering $q=0.9345$, admissible range of Jacobi constant $C$ is $[1,2.88] . C=2.881$ gives negative $\dot{y}^{2}$ within region $x=0.937$ to 0.956 which is excluded region for infinitesimal mass as velocity becomes complex. Also, for $q=0.9645$ admissible range of Jacobi constant $C$ is $[1,2.943]$. $C=2.944$ gives negative $\dot{y}^{2}$ within region $x=0.945$ to 0.957 which is excluded region for infinitesimal mass as velocity becomes complex. It is observed that excluded region becomes smaller. It is slightly shifted towards Saturn.

PSS of island corresponding to Saturn centered periodic orbit for $C=2.8$ with $q=0.9,0.9345,0.9645$ and 1 are shown in Figures 19-22 respectively. Center of the island gives Saturn centered periodic orbit at $x=0.95285$, $x=0.72165, x=0.6365$, and at $x=0.56455$ respectively.

Figures 23-26 show PSS for island corresponding to Saturn centered periodic orbit for $C=2.79$ with $q=0.9$, $0.9345,0.9645$ and 1 respectively. Center of the island gives Saturn centered periodic orbit at $x=0.8957$, $0.70345,0.62315$ and at 0.55435 respectively.

Figures 27-30 depict PSS of island for $C=2.78$ with $q=0.9,0.9345,0.9645$ and 1 respectively. Center of the island gives Saturn centered periodic orbit at $x=0.8429,0.68645,0.61065$, and at $x=0.5444$ respectively. From this it is observed that for a given $C$ by decreasing perturbation due to solar radiation pressure Saturn centered periodic orbit moves towards Sun. This is the effect of solar radiation pressure on retrograde Saturn centered periodic orbits. From Figure 19, Figure 23 and Figure 27 it can be observed that for $q=0.9$, by decreasing Jacobi constant $C$, Saturn centered periodic orbit moves towards Sun. Similar results can be observed in Figure 20, Figure 24 and Figure 28 for $q=0.9345$, in Figure 21, Figure 25 and Figure 29 for $q=0.9645$ and in Figure 22, Figure 26 and Figure 30 for $q=1$. This is the effect of Jacobi constant on Saturn centered retrograde periodic orbits. The shifting of Saturn centered retrograde periodic orbits towards Saturn by increasing Jacobi constant $C$ is plotted as dark line in Figures 31-35 for $q=1, q=0.9845, q=0.9645, q=0.9345, q=0.9$ respectively. The increase in diameter associated with a decrease in the Jacobi constant $C$ is plotted by dotted line. It should be noted that for plotting graphs in Figures 31-36 the same scale is used.

Figure 36 showing the variation of location of Saturn centered periodic orbit with variation in perturbation due to solar radiation pressure $q$ for $C=2.8, C=2.795, C=2.79, C=2.785, C=2.78$. It is clear from Figure 36 for given $C$ by reducing perturbation due to solar radiation pressure location of periodic orbit moves towards 


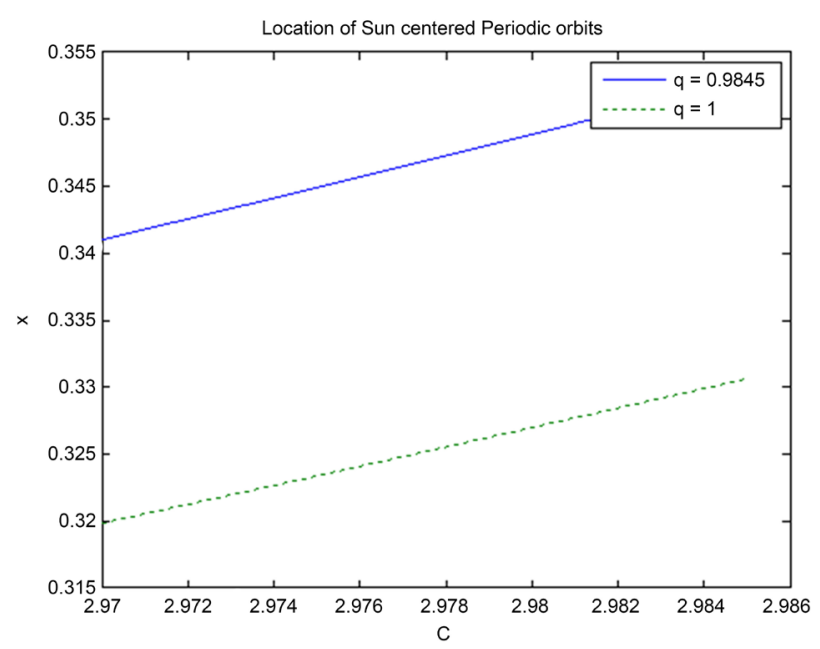

Figure 16. Variation in location of periodic orbit for $q=0.9845$ and $q=1$.

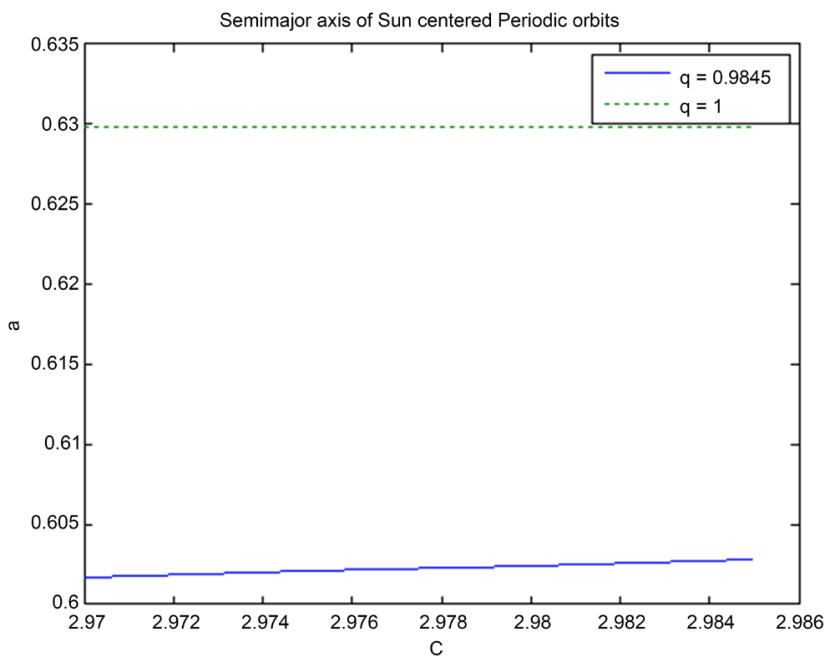

Figure 17. Variation of semi major axis for $q=0.9845$ and $q=1$.

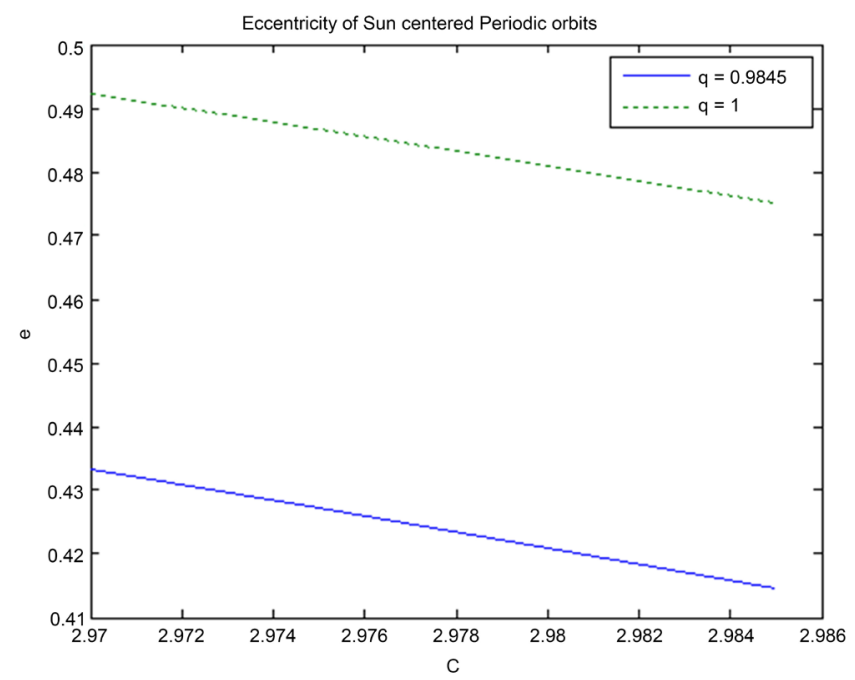

Figure 18. Variation of eccentricity for $q=0.9845$ and $q=1$. 


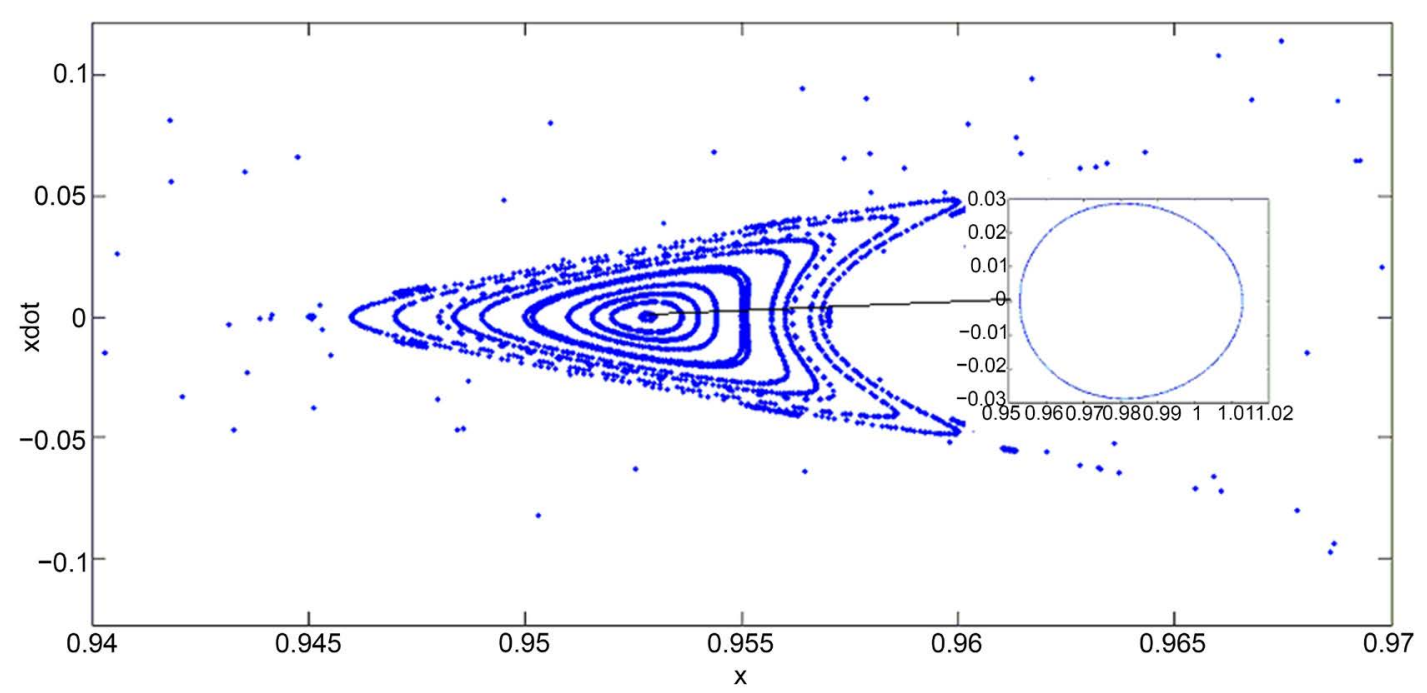

Figure 19. $C=2.8, q=0.9$.

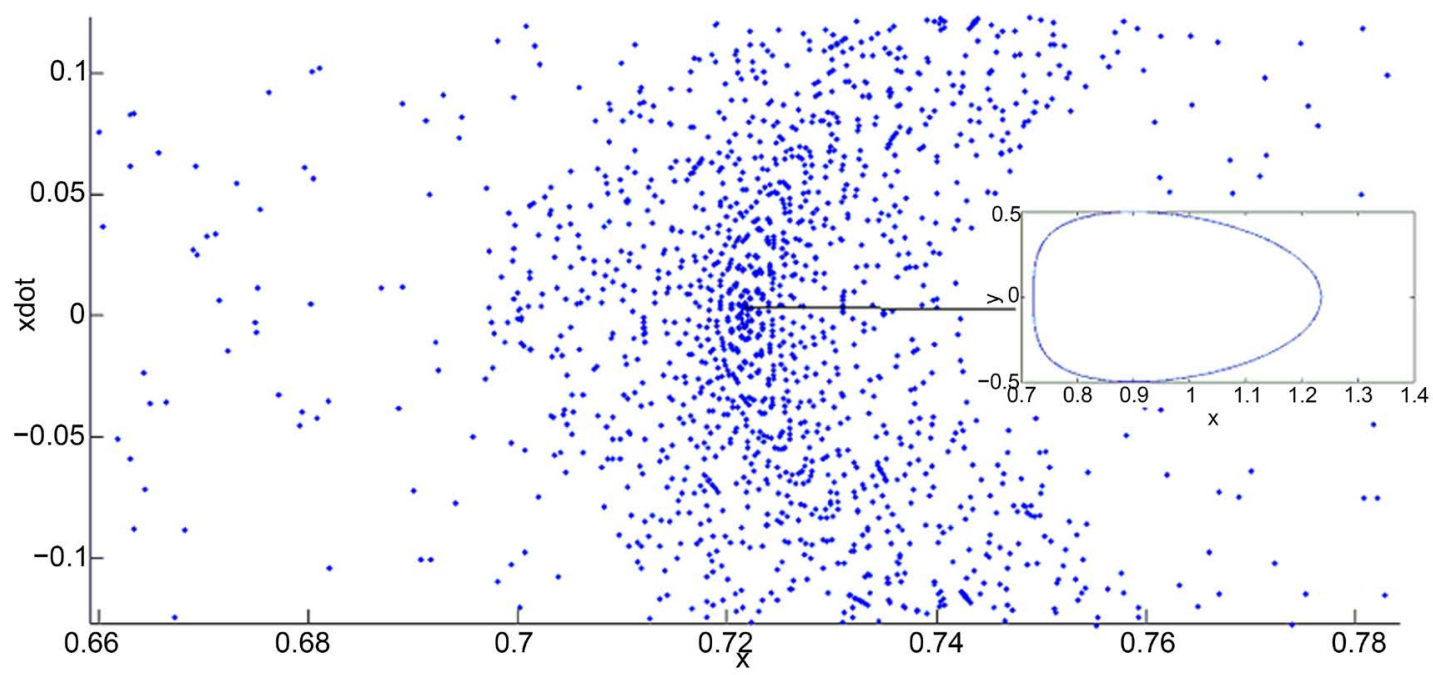

Figure 20. $C=2.8, q=0.9345$.

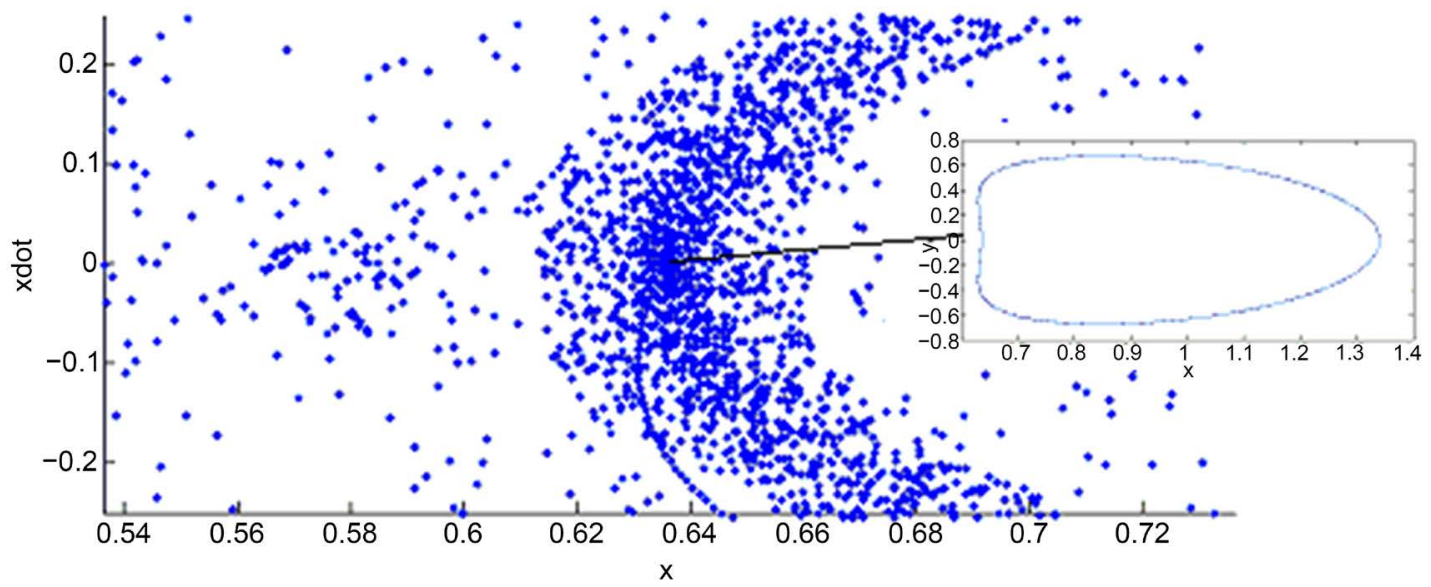

Figure 21. $C=2.8, q=0.9645$. 


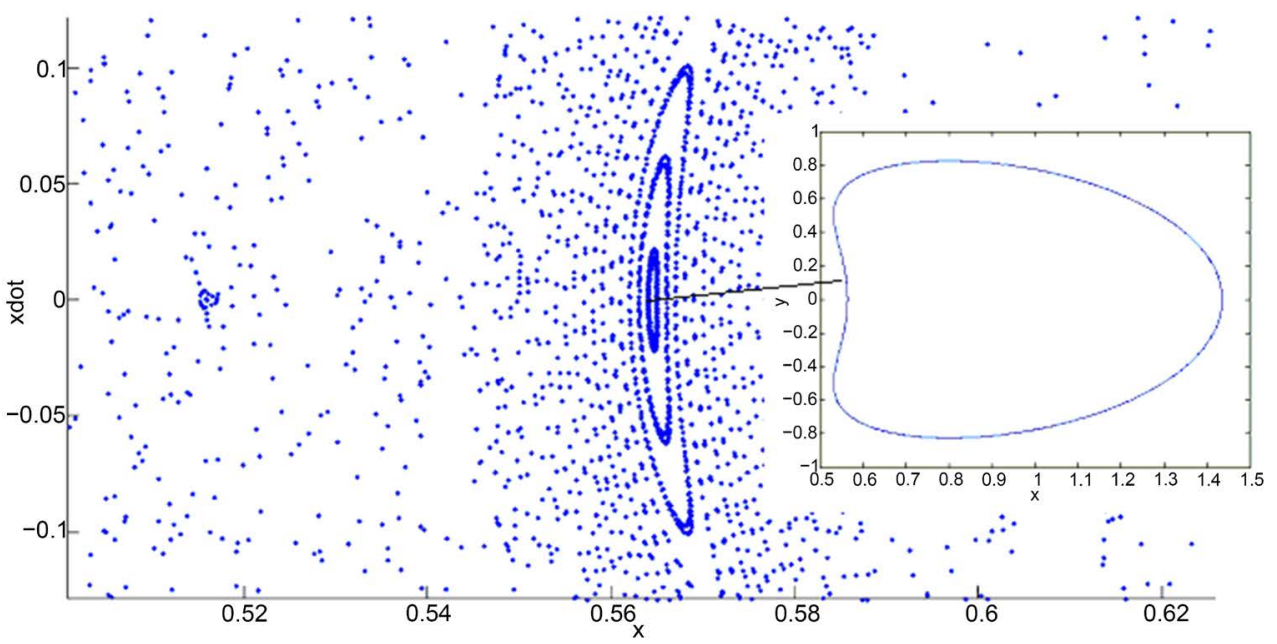

Figure 22. $C=2.8, q=1$.

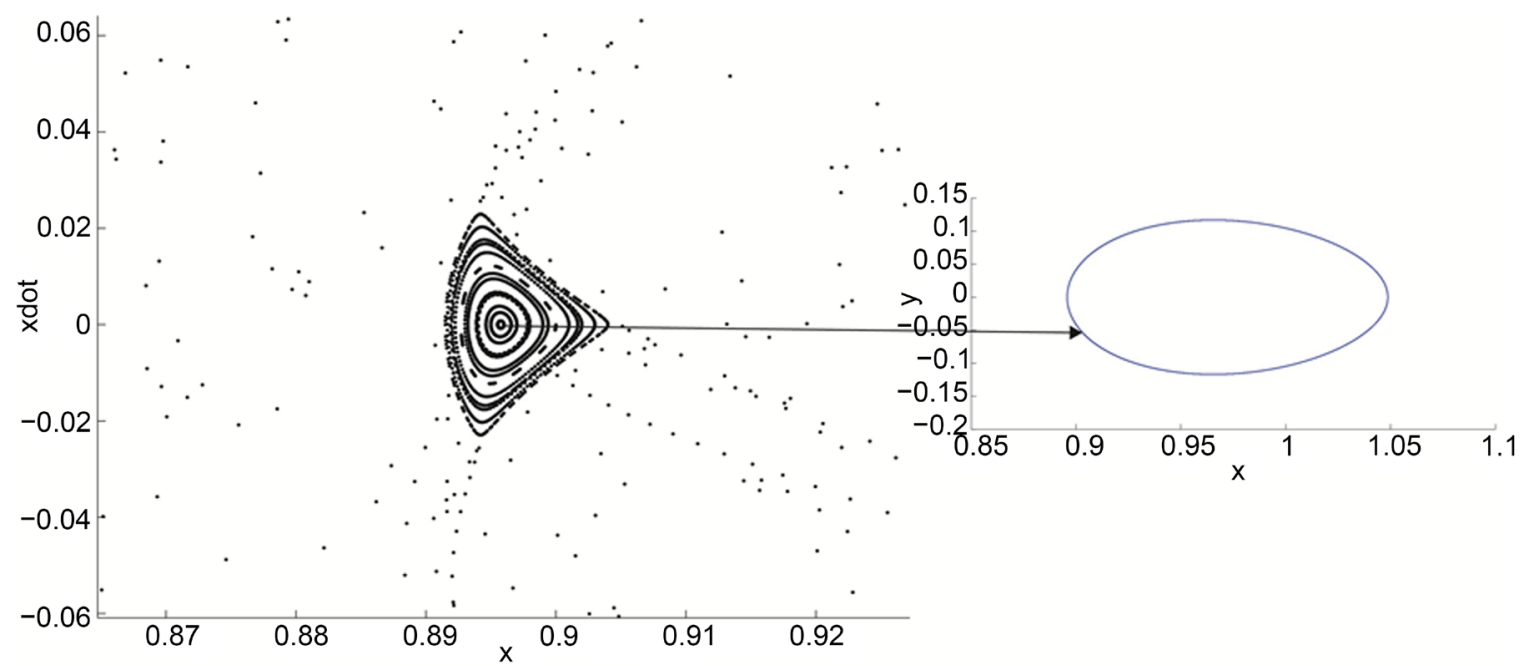

Figure 23. $C=2.79, q=0.9$.

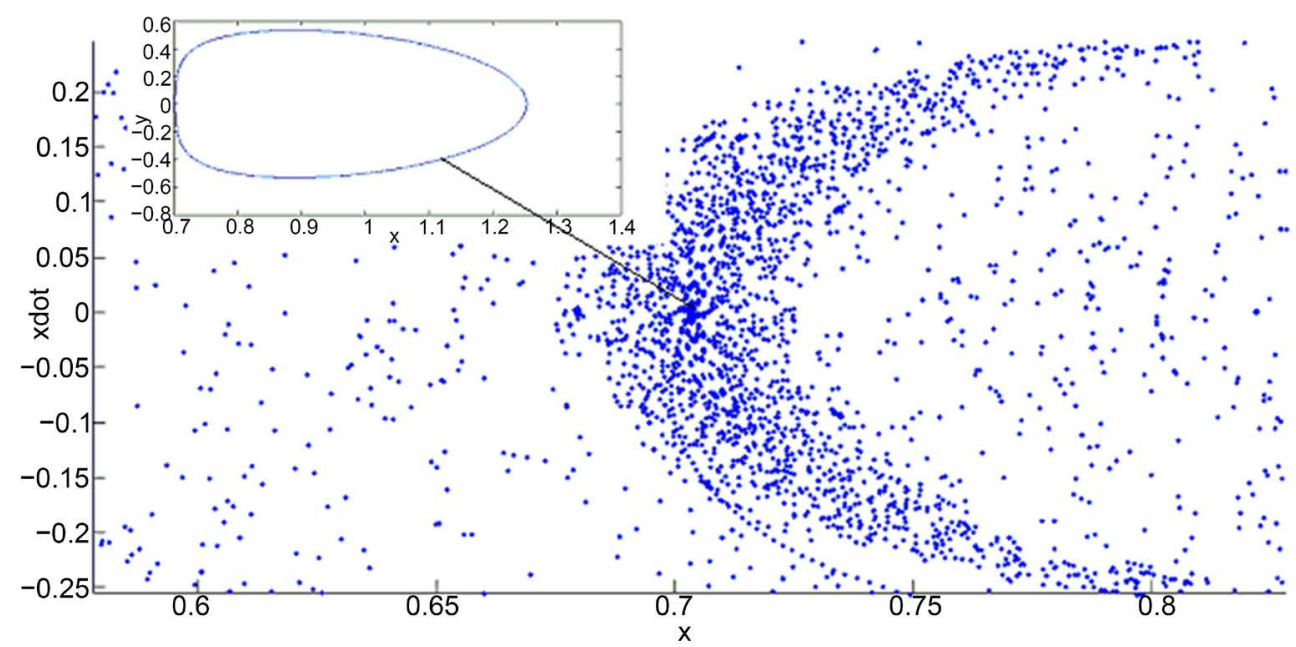

Figure 24. $C=2.79, q=0.9345$. 


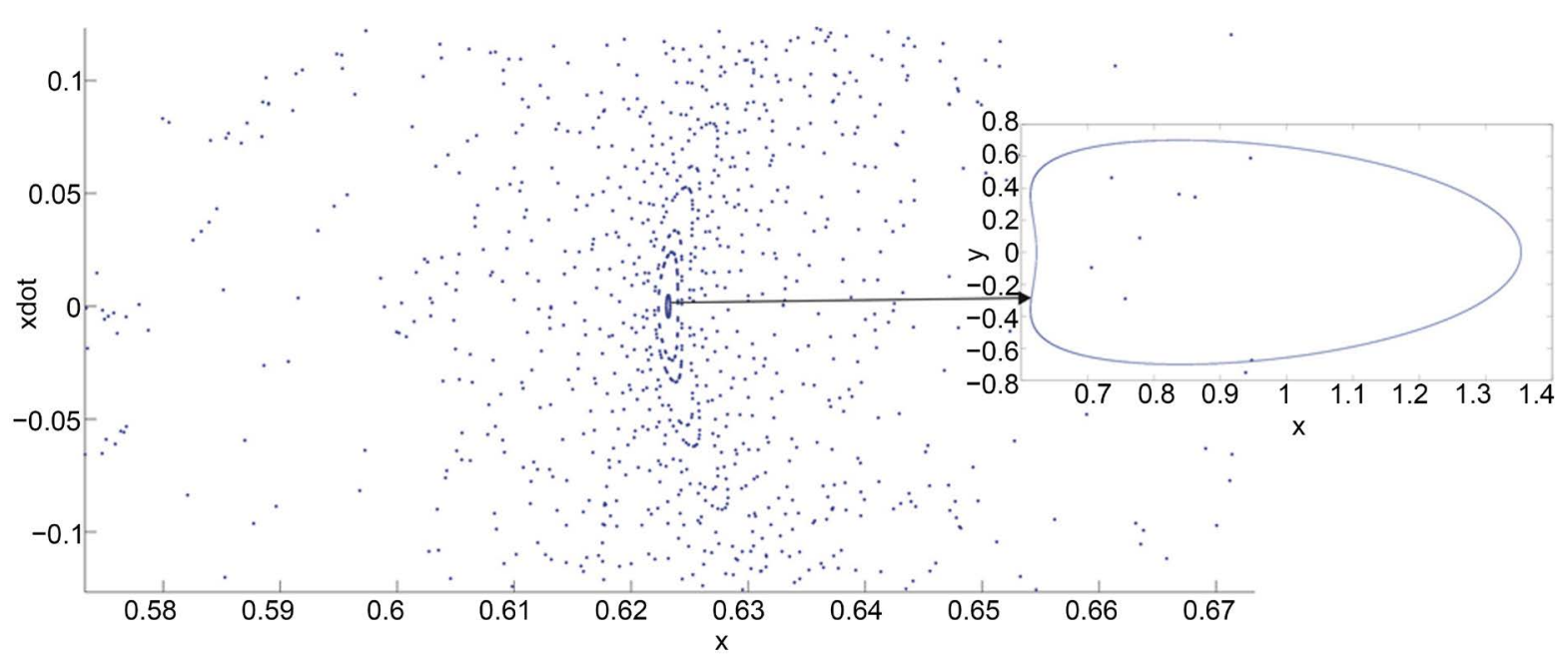

Figure 25. $C=2.79, q=0.9645$.

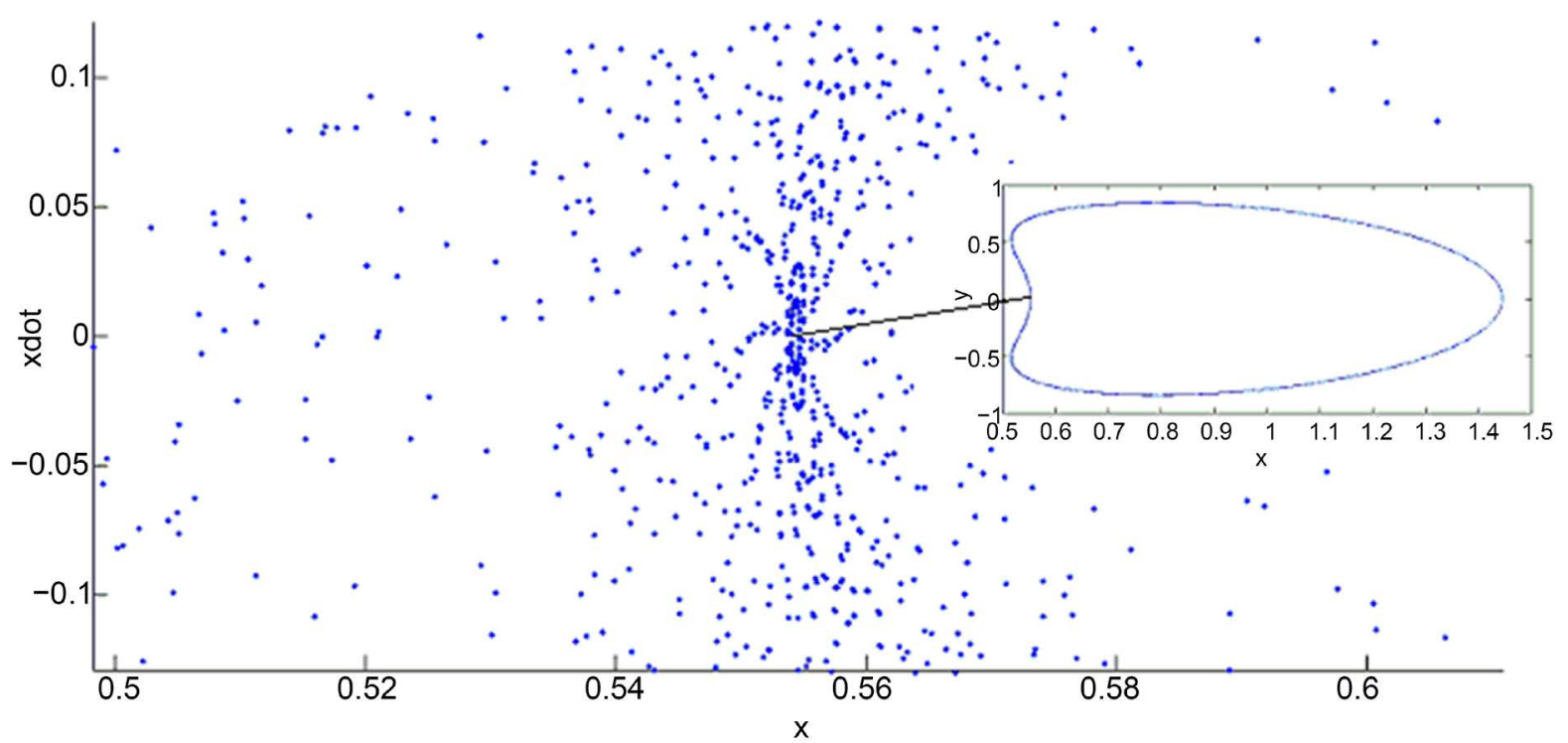

Figure 26. $C=2.79, q=1$.

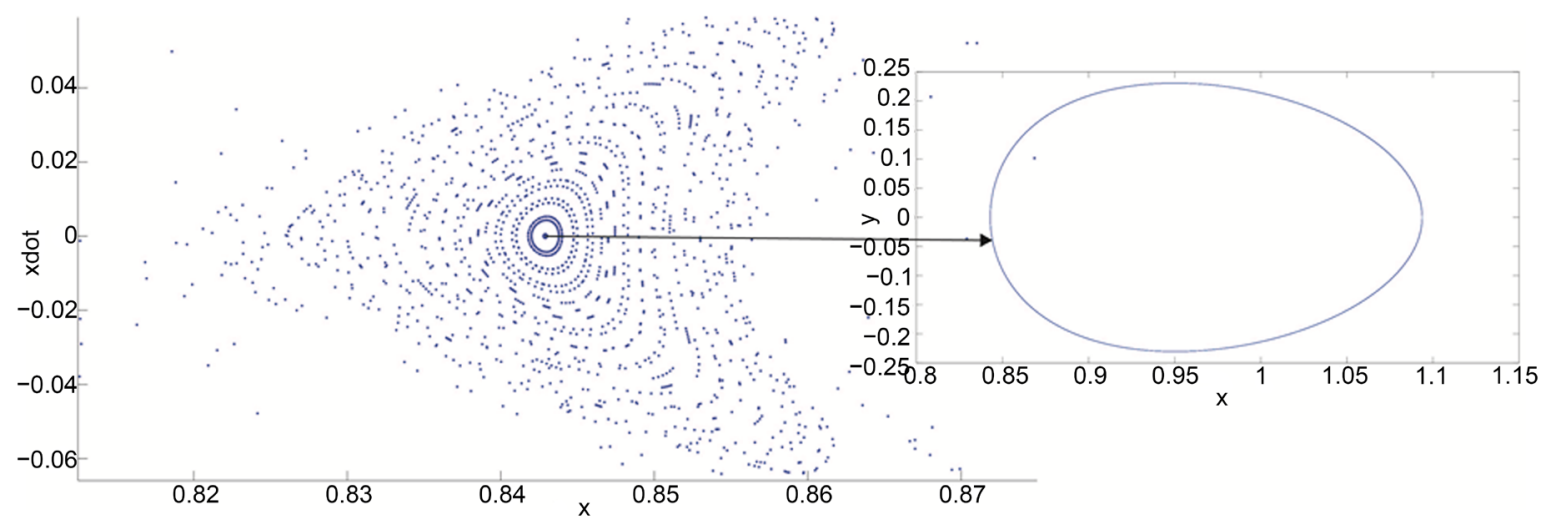

Figure 27. $C=2.78, q=0.9$. 


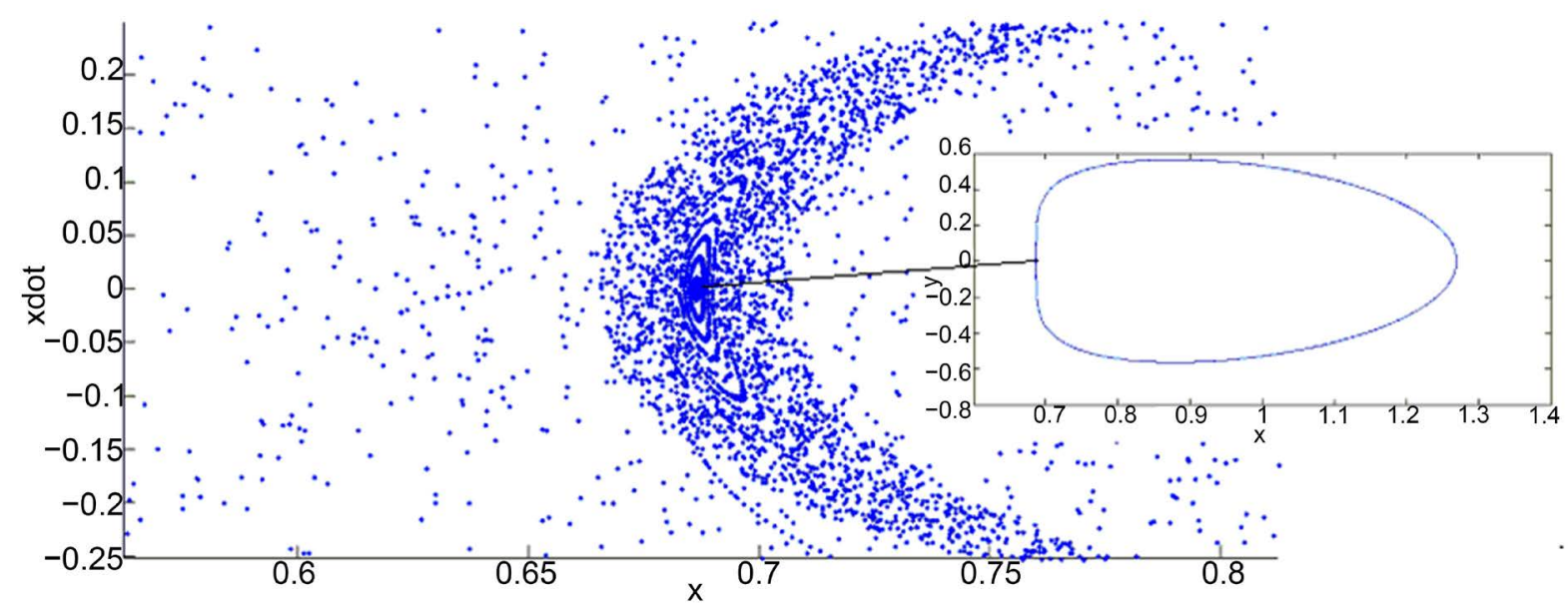

Figure 28. $C=2.78, q=0.9345$.

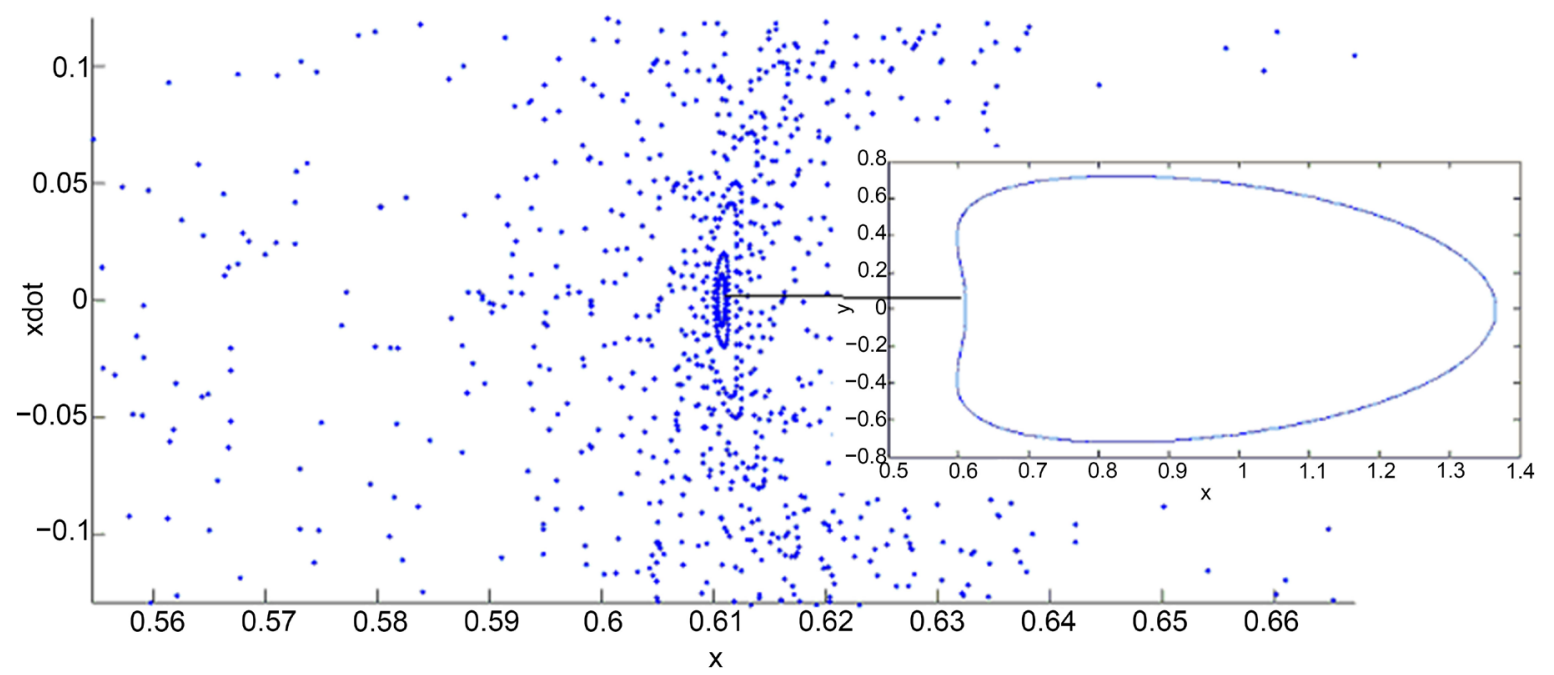

Figure 29. $C=2.78, q=0.9645$.

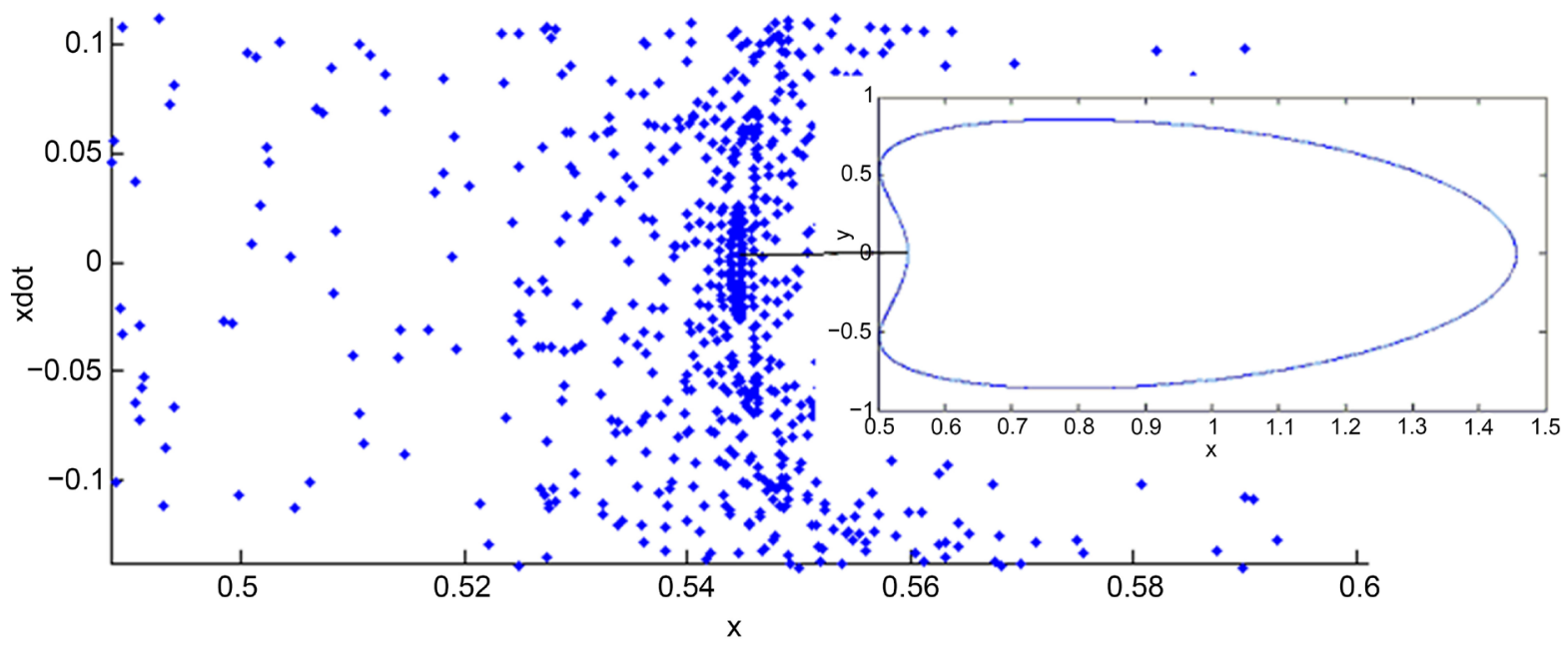

Figure $30 . C=2.78, q=1$. 


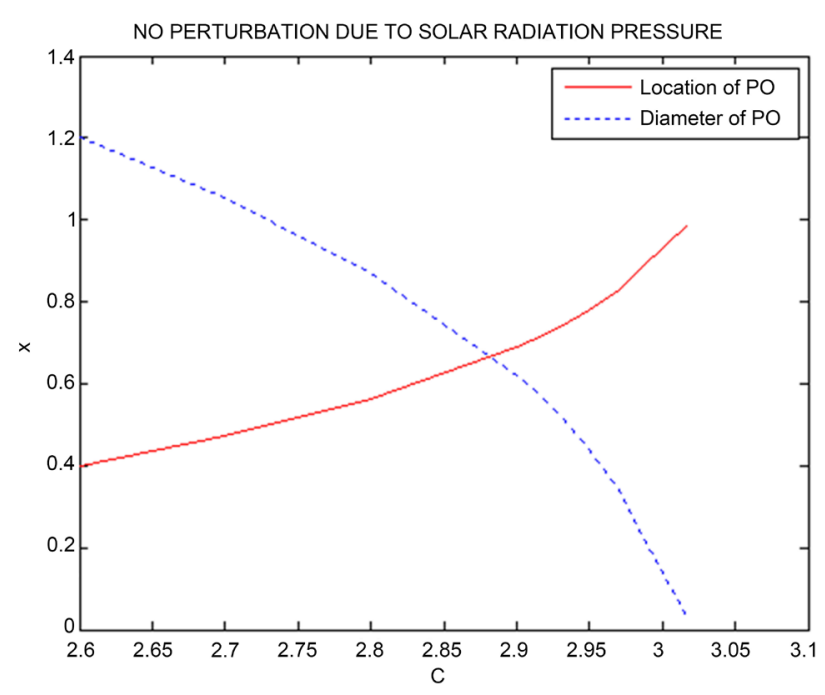

Figure 31. Variation in location and diameter for $q=1$.

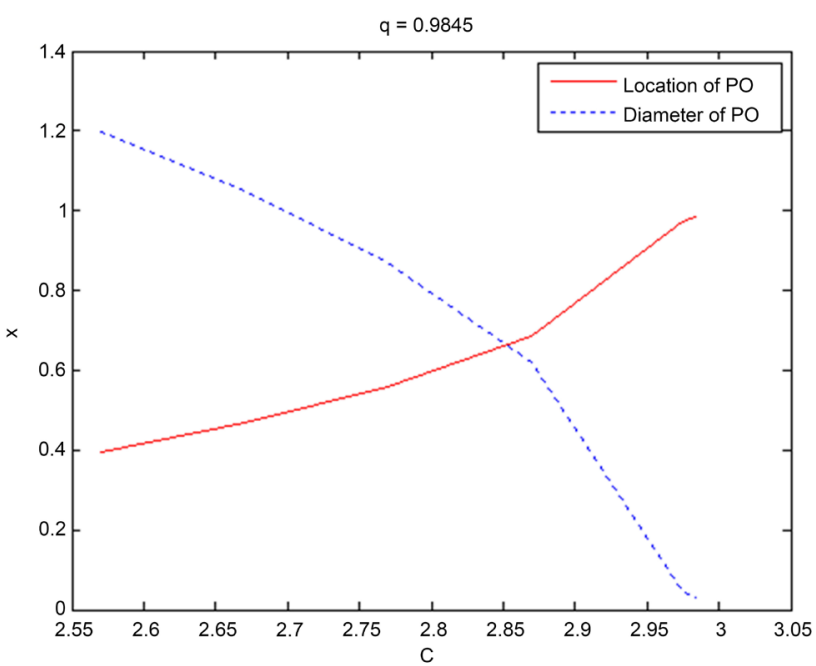

Figure 32. Variation in location and diameter for $q=0.9845$.

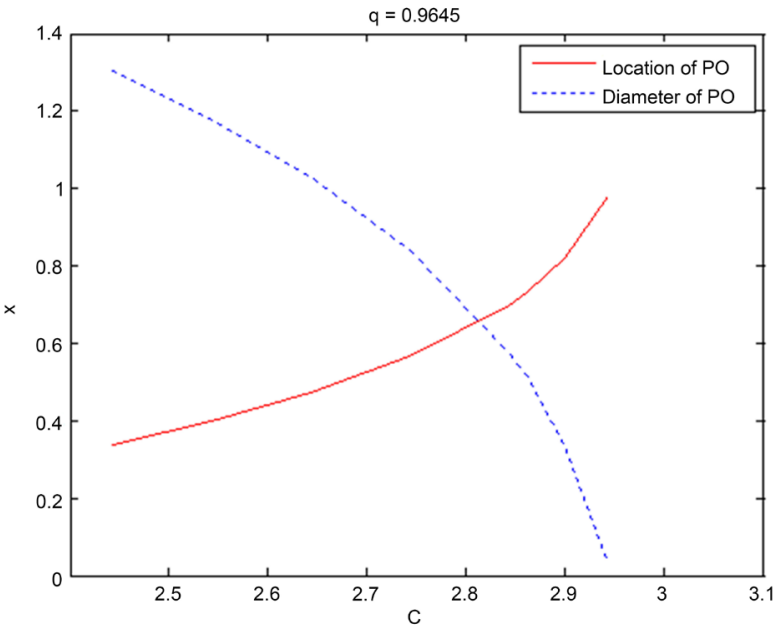

Figure 33. Variation in location and diameter for $q=0.9645$. 


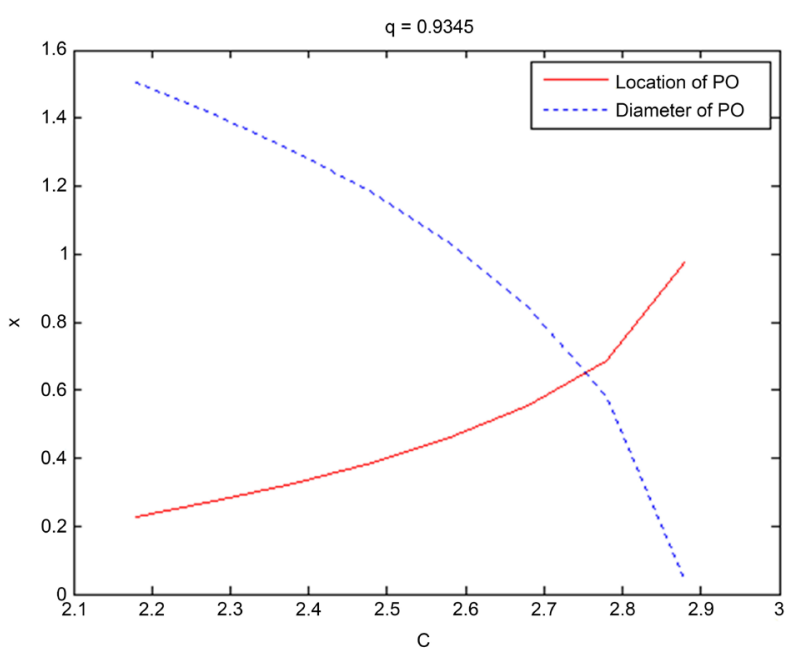

Figure 34. Variation in location and diameter for $q=0.9345$.

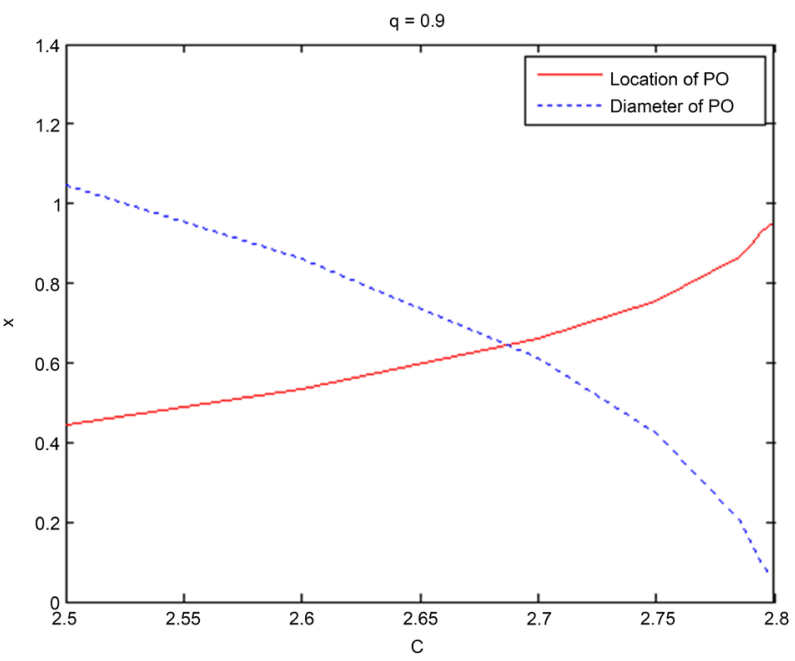

Figure 35. Variation in location and diameter for $q=0.9$.

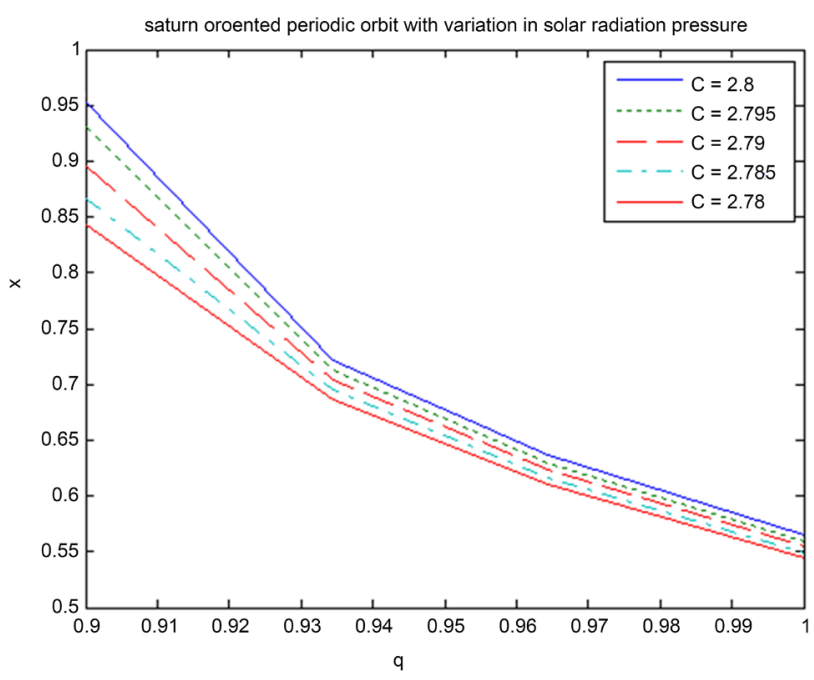

Figure 36. Variation in location of periodic orbit for $q$. 
Sun which is similar to Sun centered periodic orbits.

Figure 37 depicts the variation of semi major axis without perturbation due to solar radiation pressure. It is clear from the graph that as $C$ increases semi major axis increases. Sudden change in semi major axis is seen between $C=2.985$ and $C=3.018$. For $C=2.985$ semi major axis of periodic orbit has value 1.0234 and for $C=$ 3.018 semi major axis of periodic orbit is 1.43022 . Figure 38 showing variation of semi major axis for $q=$ 0.9845 . Observable change in semi major axis is between $C=2.87$ and $C=2.97$. For $C=2.87$ semi major axis of Saturn centered periodic orbit is 0.97012 and for $C=2.97$ it is 1.1171 . Again sudden change in semi major axis is between $C=2.97$ and $C=2.985$. Semi major axis for $C=2.985$ is 1.3269 .

Figure 39 showing variation of semi major axis for $q=0.9645$. Sudden change in the semi major axis between $C=2.9$ and $C=2.943$. For $C=2.9$ semi major axis for Saturn centered periodic orbit is 0.9519 whereas for $C=2.943$ it is 1.2328 . Figure 40 depicts the variation of semi major axis for $q=0.9345$. Observable change is between $C=2.78$ and $C=2.88$. For $C=2.78$ semi major axis of Saturn centered periodic orbit is 0.8754 and for $C=2.88$ it is 1.2109 .

Figure 41 shows uniform change in semi major axis of Saturn centered periodic orbits. From Figures 37-41 it is observed that as $q$ moves from 1 to 0.9 , sudden change in semi major axis of Saturn centered periodic orbit

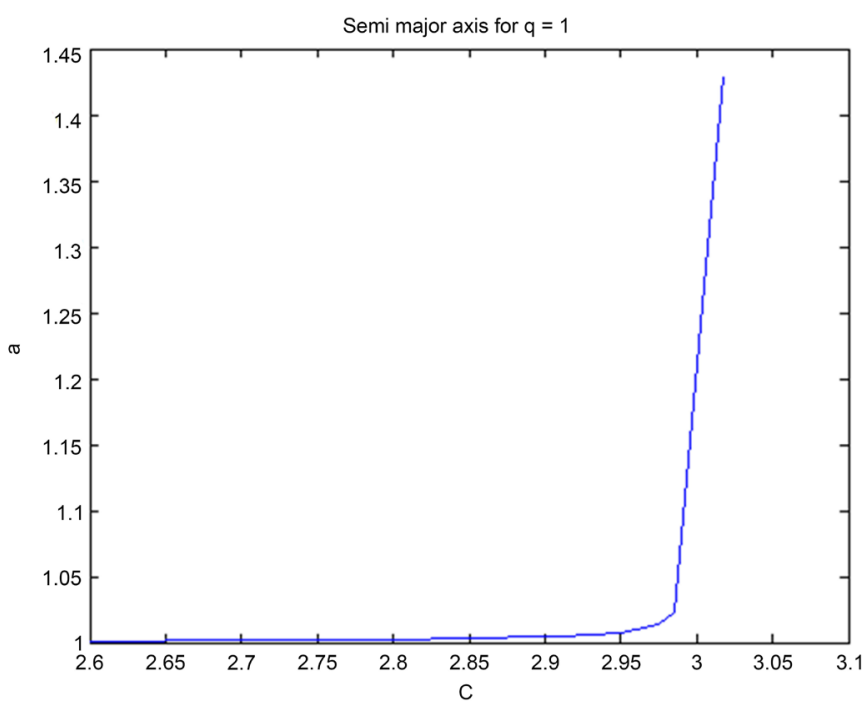

Figure 37. Semi major axis for $q=1$.

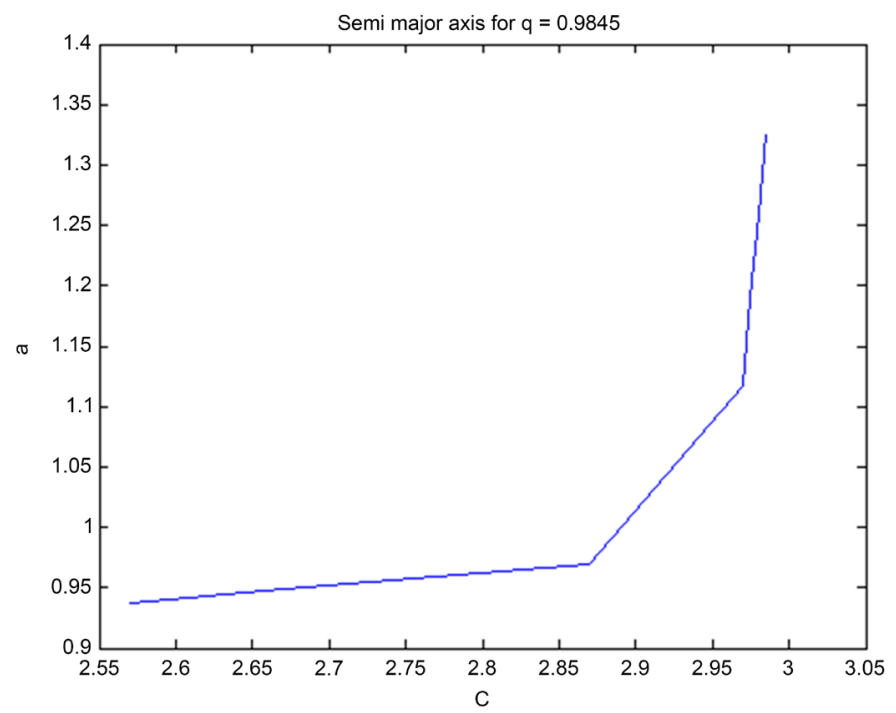

Figure 38. Semi major axis for $q=0.9845$. 


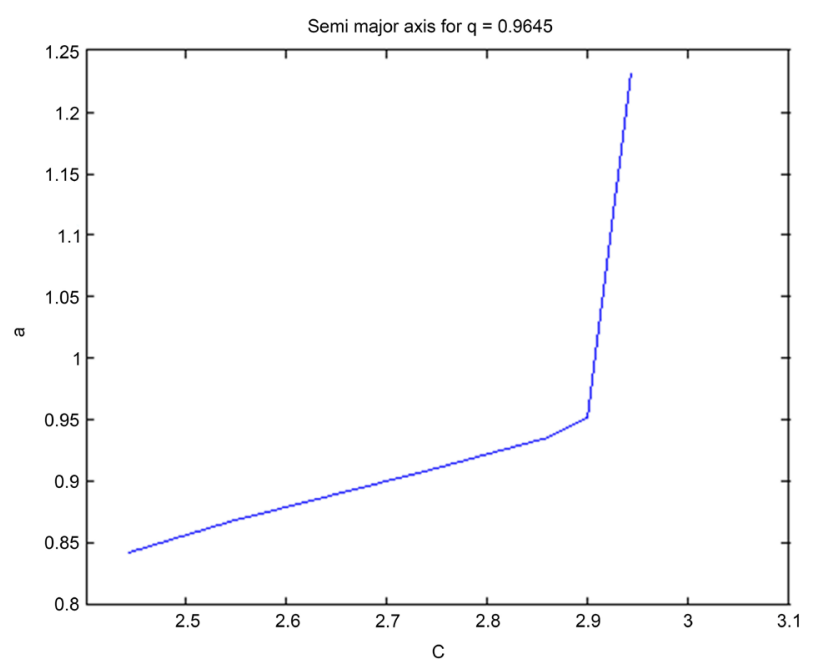

Figure 39. Semi major axis for $q=0.9645$.

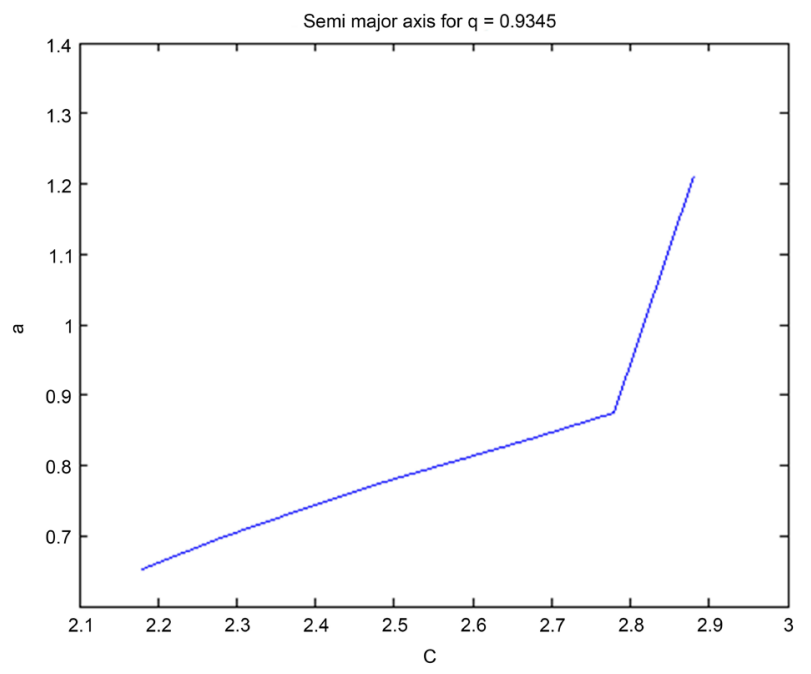

Figure 40. Semi major axis for $q=0.9345$.

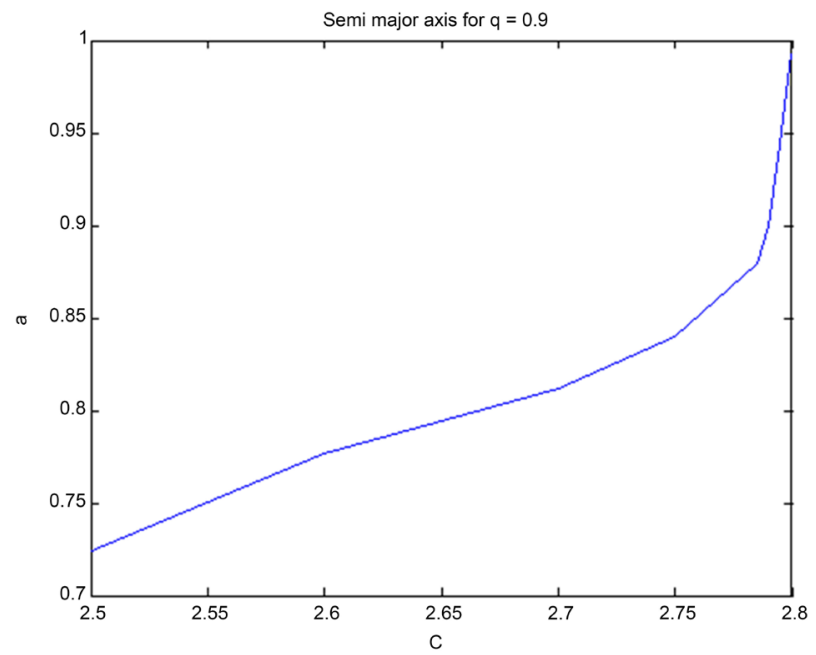

Figure 41. Semi major axis for $q=0.9$. 
becomes slow and smooth change in semi major axis. Figure 42 shows the variation of eccentricity of Saturn centered periodic orbit for $q=1$ that is without perturbation due to solar radiation pressure. It is clear from the graph as $C$ increases eccentricity decreases. But the sudden change is between $C=2.985$ and $C=3.018$. For $C=$ 2.985 eccentricity is 0.14036 and for $C=3.018$ it is 0.31045 . It is the same $C$ for which Figure 37 of semi major axis showing sudden change.

Figure 43 shows the variation of eccentricity of Saturn centered periodic orbit for $q=0.9845$. It is clear from Figure 43 that as $C$ increases eccentricity decreases. But the sudden change is between $C=2.975$ and $C=2.98$. For $C=2.975$ eccentricity is 0.1834 and for $C=2.98$ it is 0.2226 . Also for $C=2.985$ it is 0.25884 . It is for the same value of $C$ for which Figure 38 shows the sudden change in semi major axis. Figure 44 shows the variation of eccentricity of Saturn centered periodic orbit for $q=0.9645$. It is clear from the graph that as $C$ increases eccentricity decreases. But the sudden change occurs between $C=2.9$ and $C=2.943$. For $C=2.9$ eccentricity is 0.1376 and for $C=2.943$ it is 0.2051 . As earlier, it is for the same value of $C$ for which Figure 39 shows the sudden change in semi major axis. Figure 45 shows the variation of eccentricity of Saturn centered periodic orbit for $q=0.9345$. It is clear from the graph that as $C$ increases eccentricity decreases. Figure 46 shows the variation of eccentricity of Saturn centered periodic orbit for $q=0.9$. It is clear from the graph that when $C$ increases eccentricity decreases. But in this case the sudden change in the graph occurs between $C=2.795$ and $C=2.8$. For $C=2.795$ eccentricity is 0.0274 and for $C=2.8$ it is 0.0469 . It is for the same value of $C$ for which

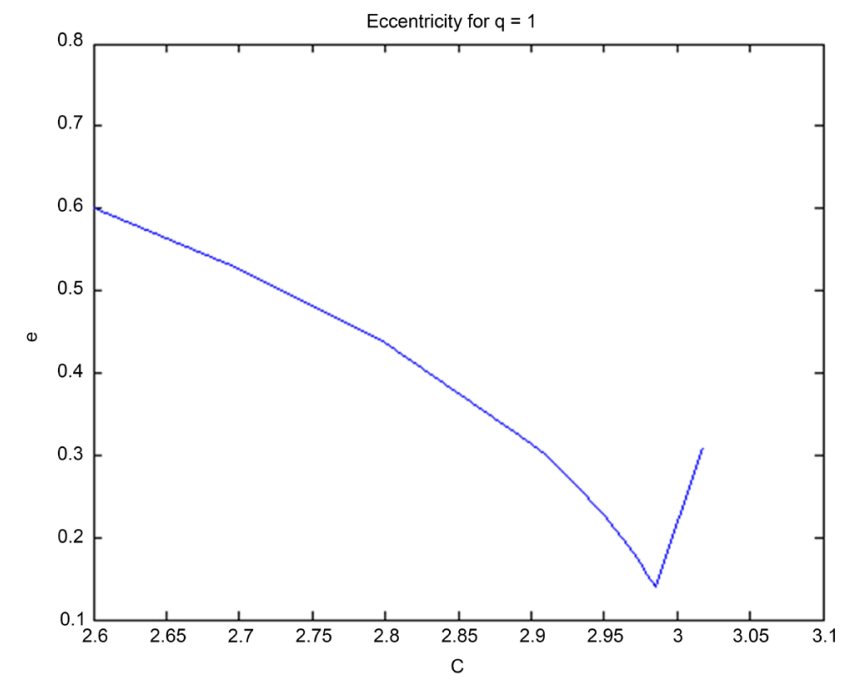

Figure 42. Eccentricity for $q=1$.

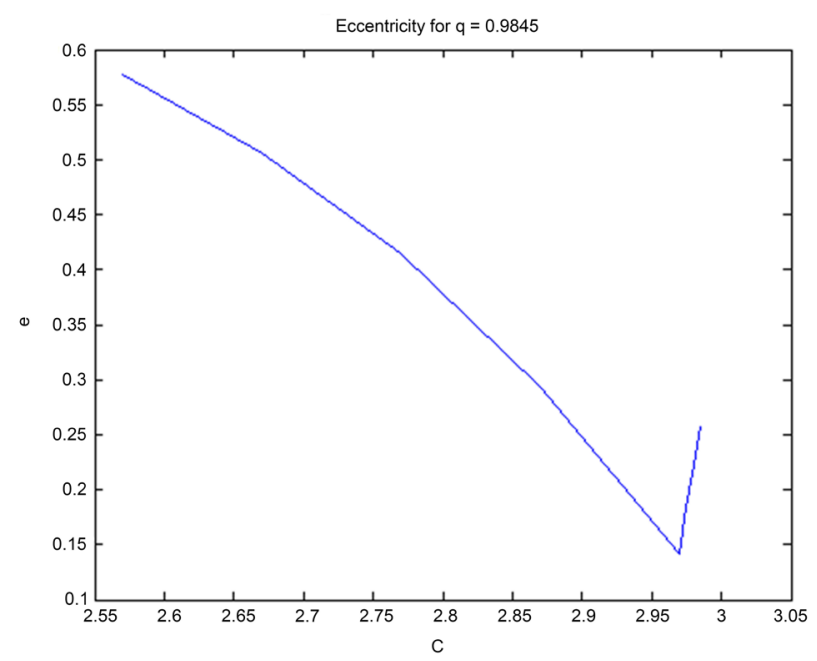

Figure 43. Eccentricity for $q=0.9845$. 


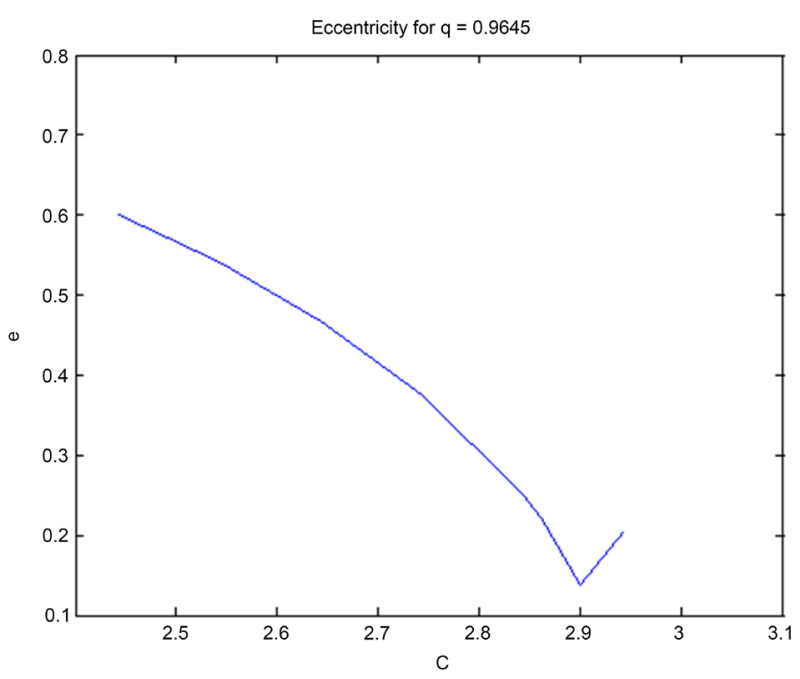

Figure 44. Eccentricity for $q=0.9645$.

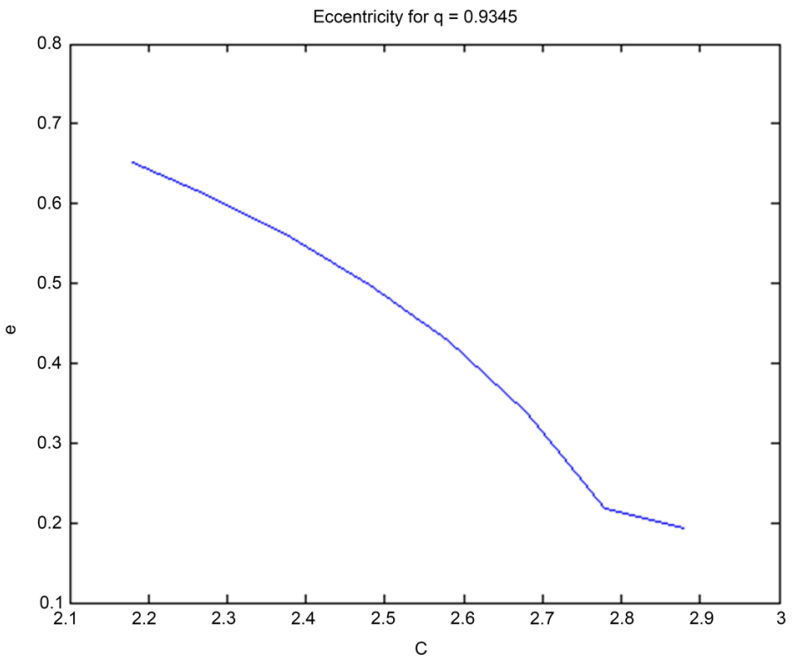

Figure 45. Eccentricity for $q=0.9345$.

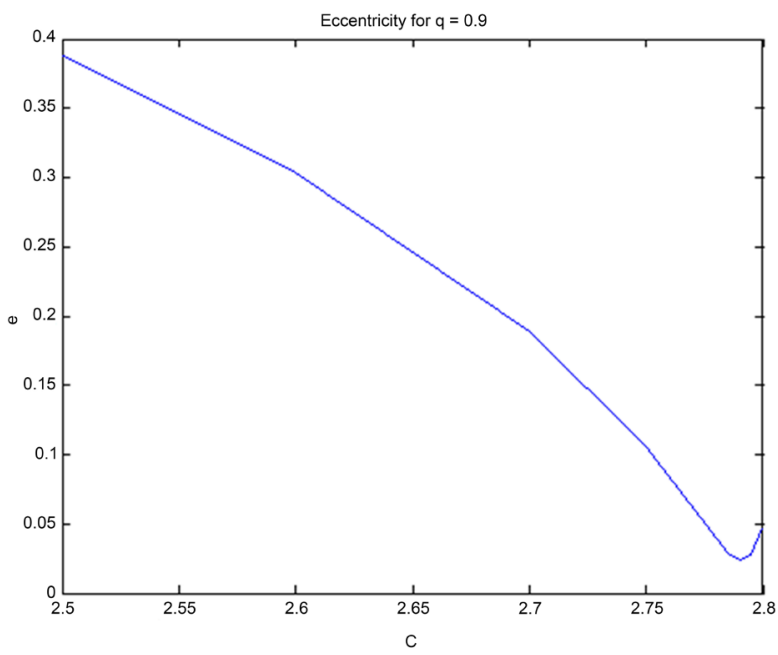

Figure 46. Eccentricity for $q=0.9$. 
Figure 41 showed the sudden change in semi major axis. From Figures 42-46, it is concluded that as q moves from 1 to 0.9 , eccentricity of Saturn centered periodic orbits decreases slowly up to certain limit of $C$ and then increases. It means perturbation due solar radiation pressure slows down the tendency of quick change in semi major axis and eccentricity against Jacobi constant $C$.

\section{Conclusion}

It is observed that as the Jacobi constant $C$ increases, the number of islands, number of quasi periodic orbits and number of periodic orbits increases for any perturbation due to solar radiation pressure. As q tends to 1 , admissible range of Jacobi constant $C$ increases. In other words, solar radiation pressure reduces admissible range of $C$. For $q=1$ maximum value of $C$ is 3.018. By increasing perturbation due to solar radiation pressure up to $q=$ 0.9845 maximum value of Jacobi constant reaches $C=2.985$, by increasing more perturbation due to solar radiation pressure up to $q=0.9645$ maximum value of $C$ decreases and reach at $C=2.943$. For $q=0.9345$, maximum value of $C$ reaches at $C=2.88$ and for $q=0.9$ maximum value of $C$ is 2.807 . It is further noticed that as Jacobi constant $C$ increases Sun centered periodic orbit and Saturn centered periodic orbits shift towards Saturn for a given perturbation due to solar radiation pressure $q$. It means Jacobi constant $C$ acting opposite to gravitational force. For a given $C$, as $q$ tends to 1, location of Sun centered and Saturn centered periodic orbits moves towards Sun. That is solar radiation pressure is responsible for shifting periodic orbits towards Saturn which is expected as solar radiation pressure is opposite to gravitational attraction of Sun. Thus, by decreasing perturbation due to solar radiation pressure, effect of gravitational attraction increasing and as a result periodic orbit shift towards Sun. For a given $q$, as $C$ increases semi major axis increases and eccentricity of Sun centered periodic orbits decreases. For a given $q$ and its corresponding maximum value of Jacobi constant $C$ Saturn centered periodic orbit showing sudden change in semi major axis and eccentricity. It is observed that semi major axis increases uniformly up to certain value of $C$ and then shows a sharp increase, where as eccentricity decreases uniformly up to certain value of $C$ and then shows a sharp increase. When the solar radiation pressure $q$ tends to 0.9 , this sharp change in the semi major axis and eccentricity graphs becomes smooth. Thus, it is concluded that perturbation due to solar radiation pressure reduces sudden change in semi major axis and eccentricity of Saturn centered periodic orbits. It is further observed that as $q$ tends to 1 , semi major axis and eccentricity of Sun centered periodic orbit increases for given $C$. In other words, due to solar radiation pressure, semi major axis and eccentricity of Sun centered periodic orbit reduces.

\section{References}

[1] Szebehely, V. (1967) Theory of Orbits. Academic Press, San Diego.

[2] Poynting, J.H. (1904) Radiation in the Solar System: Its Effect on Temperature and its Pressure on Small Bodies. Philosophical Transactions of the Royal Society A: Physical Sciences and Engineering, 202, 525-552. http://dx.doi.org/10.1098/rsta.1904.0012

[3] Robertson, H.P. (1937) Dynamical Effects of Radiation in the Solar System. Monthly Notices of the Royal Astronomical Society, 97, 423-438. http://dx.doi.org/10.1093/mnras/97.6.423

[4] Abouelmagd, E.I. (2013) The Effect of Photogravitational Force and Oblateness in the Perturbed Restricted ThreeBody Problem. Astrophysics and Space Science, 346, 51-69. http://dx.doi.org/10.1007/s10509-013-1439-9

[5] Broucke, R.A. (1968) Periodic Orbits in the Restricted Three-Body Problem with Earth-Moon Masses. Technical Report, No. 32, Jet Propulsion Laboratory, Pasadena.

[6] Poincare, H. (1892) Les Methodes Nouvelles de la Mechanique Celeste. Vol. 1, Gauthier-Villas, Paris, 82.

[7] Murray, C.D. and Dermot, S.F. (1999) Solar System Dynamics. Cambridge University Press, Cambridge.

[8] Kolmen, E., Kasdin, N.J. and Gurfil, P. (2007) Quasi-Periodic Orbits of the Restricted Three-Body Problem Made Easy. New Trends in Astrodynamics and Applications III, Vol. 886, Melville, 16-18 August 2006, 68. http://dx.doi.org/10.1063/1.2710044

[9] Winter, O.C. (2000) The Stability Evolution of a Family of Simply Periodic Lunar Orbits. Planetary and Space Science, 48, 23-28. http://dx.doi.org/10.1016/S0032-0633(99)00082-3

[10] Dutt, P. and Sharma, R.K. (2010) Analysis of Periodic and Quasi-Periodic Orbits in the Earth-Moon System. Journal of Guidance, Control, and Dynamics, 33, 1010-1017. http://dx.doi.org/10.2514/1.46400

[11] Dutt, P. and Sharma, R.K. (2011) Evolution of Periodic Orbits in the Sun-Mars System. Journal of Guidance, Control, and Dynamics, 34, 635-644. http://dx.doi.org/10.2514/1.51101 
[12] Safiya Beevi, A. and Sharma, R.K. (2012) Oblateness Effect of Saturn on Periodic Orbits in the Saturn-Titan Restricted Three-Body Problem. Astrophysics and Space Science, 340, 245-261. http://dx.doi.org/10.1007/s10509-012-1052-3

[13] Safiya Beevi, A. (2012) Study of Orbits in the Restricted Three Body Problem When More Massive Primary Is an Oblate Spheroid. Ph.D. Thesis, University of Kerala, VSSC, Thiruvananthapuram.

[14] Sharma, R.K. and Subba Rao, P.V. (1976) Stationary Solutions and Their Characteristic Exponents in the Restricted Three-Body Problem When the More Massive Primary Is an Oblate Spheroid. Celestial mechanics, 13, 137-149. http://dx.doi.org/10.1007/BF01232721

[15] Sharma, R.K. (1987) The Linear Stability of Libration Points of the Photogravitational Restricted Three-Body Problem when the Smaller Primary Is an Oblate Spheroid. Astrophysics and Space Science, 135, 271-281. http://dx.doi.org/10.1007/BF00641562 